\title{
Modeling of tension-compression asymmetry and orthotropy on metallic materials: Numerical implementation and validation
}

\author{
P.D. Barros ${ }^{\mathrm{a}, *}$, J.L. Alves ${ }^{\mathrm{b}}$, M.C. Oliveira ${ }^{\mathrm{a}}$, L.F. Menezes ${ }^{\mathrm{a}}$ \\ a CEMUC, Department of Mechanical Engineering, University of Coimbra, Pólo II, Rua Luís Reis Santos, Pinhal de Marrocos, 3030 -788 Coimbra, Portugal \\ ${ }^{\mathrm{b}}$ CMEMS, Microelectromechanical Systems Research Unit, University of Minho, Campus de Azurém, 4800-058 Guimarães, Portugal
}

\section{A R T I C L E I N F O}

\section{Article history:}

Received 22 January 2016

Received in revised form

9 May 2016

Accepted 23 May 2016

Available online 24 May 2016

Keywords:

Tension-compression asymmetry

Yield criterion

Sheet metal forming

Finite element implementation

СРВ06

DD3IMP

\begin{abstract}
A B S T R A C T
The details concerning the implementation of the yield criterion developed by Cazacu et al. 2006 (CPB06) [1], which accounts for both tension-compression asymmetry and orthotropy of the plastic flow, in the fully implicit FE solver DD3IMP (contraction of 'Deep Drawing 3-D IMPlicit') are presented in this work. The implemented constitutive model is extensively described, including the analytical first and second order derivatives required to the stress update algorithm. A set of anisotropy parameters describing the mechanical behavior of two metallic materials at room temperature, namely Zirconium and AZ31-Mg alloy, are identified with the DD3MAT (contraction for 'Deep Drawing 3-D MATerial') in-house code (Alves, 2004) [2]. The anisotropy parameters are identified for both the CPB06 and the Cazacu and Barlat (2001) (CB2001) [3] yield criteria, in order to emphasize the importance and role of the strength differential effect. The results clearly show that the CРB06 yield criterion is able to accurately describe both the in-plane anisotropy and tension-compression asymmetry, as well a different anisotropic behavior in uniaxial tension and uniaxial compression. The numerical simulation of a four-point bending test is performed, considering different orientations of the beam, i.e. of the hard/soft to deform direction relatively to the load direction, allowing to validate the implementation. The results obtained with the CРB06 show its ability to describe with accuracy the strain fields in the beam's central cross-section, the distribution of the tensile and compressive layers and, consequently, the shift of the neutral layer. The comparison with the results obtained with CB2001 indicates that the strength differential effect affects the final deformed shape of the beam, particularly for materials exhibiting strong tension-compression asymmetry.
\end{abstract}

c) 2016 Elsevier Ltd. All rights reserved.

\section{Introduction}

Currently, plastic forming of metals with hexagonal closed packed (HCP) structure poses tremendous challenges due to their low ductility at room temperature and their unusual deformation characteristics, i.e. very pronounced anisotropy with strong asymmetry between tensile and compressive behavior. Unlike cubic metals (both face centered cubic (FCC) and body centered cubic (BCC)), hexagonal metals deform due to the activation of mechanical twinning or non-Schmid type slip at single crystal level. In contrast to slip, twinning is a directional shear mechanism: in general, shear in one direction can produce twinning while shear in the opposite direction cannot [1,3-6]. This causes the strength differential (SD) effect, or tension-compression asymmetry. However, although less pronounced, the SD effect is also present in materials with cubic structure [7].

\footnotetext{
* Corresponding author.

E-mail address: pedro.barros@dem.uc.pt (P.D. Barros).
}

Until recent years, the SD effect has been neglected with the major developments being made in macroscopic plasticity models for materials with cubic structure. In fact, in most numerical analysis of metal forming processes, the yield surface is assumed to possess a point-symmetry with respect to the center, such that a stress state and its reverse state have the same absolute value [3,8-11].

Cazacu et al. (2004) extended an isotropic yield function to the anisotropic case through invariants generalizing, able to describe both the materials anisotropy and tension-compression asymmetry [4]. Later, Cazacu et al. presented another yield function (CPB06) that enables describing the asymmetric yielding between tension and compression due to twining as well as in-plane anisotropy through a linear transformation, with a fourth-order tensor, of the deviatoric stress tensor [1]. Some authors have later adopted several linear transformations in order to more accurately capture in-plane anisotropy [6,12]. Tuninetti et al. characterized the mechanical behavior of a Ti-6Al-4V alloy using uniaxial tension, uniaxial compression, simple shear and plane strain tests in three orthogonal material directions, identifying the CPB06 yield criterion parameters by inverse modeling of the axial strain field of 


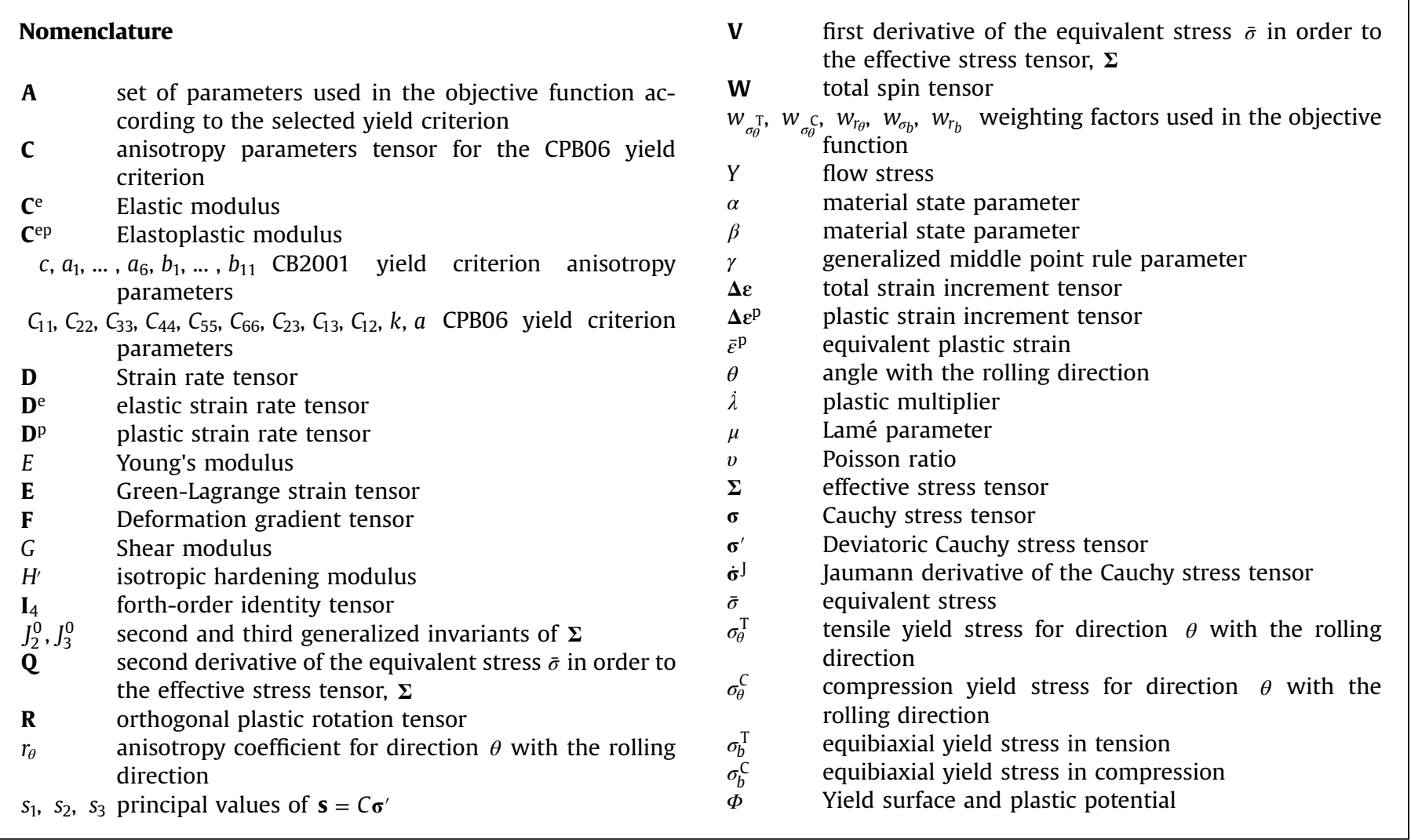

compression specimens in the three orthogonal directions of the material [13]. In fact, several works have been made for the characterization of both titanium [14,15] and magnesium alloys $[16,17]$, both presenting a HCP crystal structure.

The modeling of the SD effects allowed to point a new interpretation of Swift effects. Considering the isotropic form of the CPB06 yield criterion, Cazacu et al. showed that a slight difference between the uniaxial yield stresses in tension and compression leads to irreversible length changes under monotonic free-end testing conditions [18]. Revil-Baudard et al. show that there is a correlation between the Swift phenomenon in torsion and the stress-strain behavior in uniaxial tension and compression [17]. Chandola et al. showed that to explain and accurately predict the room-temperature torsional response of a strongly textured AZ31 $\mathrm{Mg}$ material it is necessary to account for the combined effects of anisotropy and tension-compression asymmetry at polycrystalline level [16].

Revil-Baudard and Cazacu numerically assessed the influence of the tension-compression asymmetry of the plastic flow in the matrix on void evolution and the location of the zone corresponding to maximum damage, in round tensile specimens subject to uniaxial tension [19]. Alves and Cazacu [20] used a detailed micromechanical finite-element analyses of three-dimensional unit cells, considering a spherical void at its center, with the plastic flow of the matrix described by the isotropic form of the CРB06 yield criterion. It was shown that there is a strong correlation between SD effects in the plastic flow of the matrix, arising from its dependence on the third stress invariant, with void evolution, and ultimately the ductility of porous metallic polycrystals. The combined effect of both the tension-compression asymmetry and anisotropic behavior of the material was also studied [17]. Also, due to non-negligible twinning activity accompanied by grain reorientation and highly directional grain interactions, the influence of the texture evolution on the hardening behavior of HCP materials cannot be neglected, even for the simplest monotonic loading paths [21]. In fact, Plunkett et al. shows a way of describing distortional hardening based on the evolution, with plastic strain, of the CPB06 yield locus [5].

The aim of this work is to evaluate the importance of taking into account the tension-compression asymmetry in the constitutive model. In this context, an associated flow rule is considered, neglecting the evolution of the shape of the yield surface with plastic work. The manuscript starts by presenting the details concerning the constitutive model, including the non-quadratic yield criterion proposed by Cazacu, Plunkett and Barlat and its implementation into the implicit in-house FE code DD3IMP, which has been continuously developed and optimized to simulate sheet metal forming processes $[22,23]$. In this context, the main aspects of the implementation of the СРB06 yield criterion in an implicit finite element code are presented, including the general aspects of the linear transformation operating in the deviatoric stress space. The validation of the implemented model is performed with the numerical simulation of a beam subjected to a four-point-bending test, along two different directions. The results obtained with the CPB06 yield criterion are compared with the ones of the Cazacu and Barlat (CB2001) [3] yield criterion, since the later enables an accurate description of the in-plane anisotropy while neglecting the SD effect. This allows analyzing the strain fields evolution in the beam's cross section, as well as the final shape of the beam when considering, or not, the SD effect.

\section{Constitutive model}

The constitutive equation that models the materials' mechanical behavior establishes the relationship between the most relevant state variables characterizing the continuum medium. In the following, it is assumed that constitutive modeling is 
formulated in the objective frame, and thus all tensorial quantities are invariant. The differential form of the elastoplastic behavior law is, in its general form,

$\dot{\boldsymbol{\sigma}}^{\mathrm{J}}=\mathbf{C}^{\mathrm{ep}}: \mathbf{D}$,

where $\dot{\boldsymbol{\sigma}}^{\mathrm{J}}$ is the Jaumann derivative of the Cauchy stress tensor $\boldsymbol{\sigma}$, given by

$\dot{\boldsymbol{\sigma}}^{\mathrm{J}}=\dot{\boldsymbol{\sigma}}+\boldsymbol{\sigma} \mathbf{W}-\mathbf{W} \boldsymbol{\sigma}$

where $\dot{\sigma}$ stands for the time derivative of the Cauchy stress tensor and $\mathbf{W}$ is the total spin tensor defined by

$\mathbf{W}=\dot{\mathbf{R}} \mathbf{R}^{\mathrm{T}}$,

with $\mathbf{R}$ being the orthogonal rotation tensor (it is assumed that the plastic spin tensor is negligibly small [24]). The global and objective frames are related through the rotation tensor $\mathbf{R}$, which is derived from the polar decomposition of the deformation gradient. $\mathbf{D}$ is the strain rate tensor and $\mathbf{C}^{\mathrm{ep}}$ is a fourth-order tensor corresponding to the elastoplastic modulus. The expression for this tensor depends of the algorithms adopted in the integration of the constitutive model and the type of relation considered between the states at the beginning and at the end of the loading increment. Therefore, it is possible to consider the tangent elastoplastic or the consistent elastoplastic moduli. The implicit algorithm adopted in DD3IMP relies on the Newton-Raphson method to determine the incremental displacements that guarantee the static equilibrium of the deformable body, in each increment. This method assures quadratic convergence only when the consistent elastoplastic modulus is used, i.e. when the same approximations used to calculate the incremental strains and rotations and the updated state variables are also applied to linearize the principle of virtual power [25]. The trial solution for the Newton-Raphson method is obtained using an explicit approach, relying on the tangent elastoplastic modulus, defined as [26]

$\left.\mathbf{C}^{\mathrm{ep}}\right|_{\text {tangent }}=\mathbf{C}^{\mathrm{e}}-\alpha f_{0} \mathbf{V} \otimes \mathbf{V}$,

where $\alpha=0$ if the material is in an elastic state, or during elastic unloading, and $\alpha=1$ if the material is in an elastoplastic loading state. $f_{0}$ is a function of the hardening law adopted which, considering only the isotropic hardening, can be written in the general form as

$f_{0}=\frac{4 \mu^{2}}{2 \mu \mathbf{V}: \mathbf{V}+H^{\prime}}$,

where $\mu$ is the Lamé parameter. $\mathbf{V}$ is one of the tensorial quantities to be determined as function of the adopted yield criterion, and it is, by definition

$\mathbf{V}=\frac{\partial \Phi}{\partial \boldsymbol{\sigma}^{\prime}}$,

where $\sigma^{\prime}$ is the deviatoric Cauchy stress tensor and $\Phi$ is the plastic potential, identified as a scalar potential defining the elastic limit surface, such that

$\Phi\left(\sigma^{\prime}, \bar{\varepsilon}^{\mathrm{p}}\right)=\bar{\sigma}-Y=0$,

where $\bar{\sigma}$ is the equivalent stress given by the yield criterion and $Y$ is the flow stress, modeled by the isotropic work-hardening law.

The strain rate can be decomposed into elastic and plastic parts as

$\mathbf{D}=\mathbf{D}^{\mathrm{e}}+\mathbf{D}^{\mathrm{p}}$

where $\mathbf{D}^{\mathrm{p}}$, the plastic strain rate tensor, is a deviatoric tensor, given by the associated inviscid flow rule as
$\mathbf{D}^{\mathrm{p}}=\lambda \frac{\partial \Phi}{\partial \boldsymbol{\sigma}^{\prime}}=\lambda \mathbf{V}$,

in which $\dot{\lambda}$ is the plastic multiplier. From the plastic potential definition (see (7)), the consistency condition in the rate form states that

$\dot{\Phi}(\bar{\sigma}, Y)=\dot{\bar{\sigma}}-H^{\prime} \bar{\varepsilon}^{\mathrm{p}}=0$,

where

$H^{\prime}=\partial Y / \partial \bar{\varepsilon}^{\mathrm{p}}$

is the isotropic hardening modulus. If $\Phi$ is a homogeneous function of degree one, then $\dot{\bar{\varepsilon}}^{\mathrm{p}}=\dot{\lambda}$. $\dot{\bar{\sigma}}$ and $\dot{\bar{\varepsilon}}^{\mathrm{p}}$ are, respectively, the time derivatives of the equivalent stress and of the equivalent plastic strain. The total equivalent plastic strain is defined as

$\bar{\varepsilon}^{\mathrm{p}}=\int_{0}^{t} \frac{\boldsymbol{\sigma}^{\prime}: \mathbf{D}^{\mathrm{p}}}{\bar{\sigma}} \mathrm{d} t$

\subsection{Time integration}

Since the elastic behavior is considered isotropic, the hypoelastic form of the Hooke's law can be written as [26]

$\dot{\boldsymbol{\sigma}}=\mathbf{C}^{\mathrm{e}}:\left(\mathbf{D}-\mathbf{D}^{\mathrm{p}}\right)$,

which, by integration, corresponds to the stress increment in the time interval $[t, t+\Delta t]$, i.e.

$\sigma_{\mathrm{F}}-\sigma_{0}=\mathrm{C}^{\mathrm{e}}: \Delta \boldsymbol{\epsilon}-\mathrm{C}^{\mathrm{e}}: \Delta \boldsymbol{\epsilon}^{\mathrm{p}}$,

with subscripts 0 and $\mathrm{F}$ denoting the quantities at the beginning and at the end of the time increment $[t, t+\Delta t]$, respectively. $\Delta \mathbf{\epsilon}^{\mathrm{p}}$ and $\Delta \boldsymbol{\epsilon}$ are the increments of the plastic and total strain tensors, respectively, determined over the time increment. Applying the generalized middle point rule to determine the $\mathbf{D}^{\mathrm{p}}$ evolution, and since $\mathbf{D}^{\mathrm{p}}=\dot{\lambda} \mathbf{V}$, it can be written that

$\Delta \mathbf{\epsilon}^{\mathrm{p}}=\Delta \lambda \mathbf{V}_{\gamma}$,

with

$\mathbf{V}_{\gamma}=(1-\gamma) \mathbf{V}_{0}+\gamma \mathbf{V}_{\mathrm{F}}$ and $\gamma \in[0,1]$.

To calculate $\Delta \lambda$ and $V_{\mathrm{F}}$ it is necessary to determine $\sigma_{\mathrm{F}}^{\prime}$. $\boldsymbol{\sigma}_{\mathrm{F}}$ is calculated from Eq. (14), leading to deviatoric and hydrostatic components given by, respectively,

$\boldsymbol{\sigma}_{\mathrm{F}}^{\prime}=\boldsymbol{\sigma}_{0}^{\prime}+2 G \Delta \epsilon^{\prime}-2 G \Delta \lambda \mathbf{V}_{\mathrm{F}}$ and

$\operatorname{tr}\left(\boldsymbol{\sigma}_{\mathrm{F}}\right)=\operatorname{tr}\left(\boldsymbol{\sigma}_{0}^{\prime}\right)+\frac{E}{1+2 v} \operatorname{tr}(\Delta \mathbf{\epsilon})$.

$G$ and $E$ are the shear and Young's modulus, while $v$ is the Poisson's ratio. In order to determine both $\sigma_{\mathrm{F}}^{\prime}$ and $\Delta \bar{\epsilon}^{\mathrm{p}}=\Delta \lambda$, the consistency condition at the end of the time step is imposed, i.e.

$\bar{\sigma}\left(\sigma_{F}^{\prime}\right)-Y_{F}\left(\bar{\epsilon}_{0}^{p}+\Delta \bar{\epsilon}^{p}\right)=0$.

Together, Eqs. (17) and (19) define a non-linear system of equations, which needs to be solved in the state update algorithm, with unknowns $\sigma_{F}^{\prime}$ and $\Delta \bar{\epsilon}^{p}$, i.e.,

$\mathbf{Q}\left(\boldsymbol{\sigma}_{\mathrm{F}}^{\prime}, \Delta \overline{\mathrm{e}}^{\mathrm{p}}\right)=\left[\begin{array}{l}\mathbf{G} \\ g\end{array}\right]=\left[\begin{array}{l}\mathbf{0} \\ 0\end{array}\right]$,

or

$\left[\begin{array}{c}\mathbf{G} \\ g\end{array}\right]=\left[\begin{array}{c}\boldsymbol{\sigma}_{\mathrm{F}}^{\prime}-\boldsymbol{\sigma}_{0}^{\prime}-2 G \Delta \epsilon^{\prime}-2 G \Delta \bar{\epsilon}^{\mathrm{p}} \mathbf{V}_{\mathrm{F}} \\ \bar{\sigma}\left(\boldsymbol{\sigma}_{\mathrm{F}}^{\prime}\right)-Y_{\mathrm{F}}\left(\bar{\epsilon}_{0}^{\mathrm{p}}+\Delta \bar{\epsilon}^{\mathrm{p}}\right)\end{array}\right]=\left[\begin{array}{l}\mathbf{0} \\ 0\end{array}\right]$. 
So, the updated state variables are determined solving the nonlinear system of equations Eq. (21) using the Newton-Raphson method. The calculation of $\mathbf{Q}$ and $\partial \mathbf{Q} / \partial \boldsymbol{s}$ (with $\boldsymbol{\varsigma}=\left[\boldsymbol{\sigma}_{\mathrm{F}}^{\prime}, \Delta \bar{\epsilon}^{\mathrm{p}}\right]$ ) depends on the adopted yield criterion, with $\mathbf{Q}$ calculated considering Eq. (21) and $\partial \mathbf{Q} / \partial \varsigma$ calculated considering partial derivatives, such that:

$\frac{\partial \mathbf{Q}}{\partial \boldsymbol{s}}=\left[\begin{array}{l}{[\mathbf{A}][\mathbf{c}]} \\ {[\mathbf{b}] d}\end{array}\right]$,

where

$\mathbf{A}=\frac{\partial \mathbf{Q}}{\partial \boldsymbol{\sigma}_{\mathrm{F}}^{\prime}}, \mathbf{b}=\frac{\partial \mathrm{g}}{\partial \boldsymbol{\sigma}_{\mathrm{F}}^{\prime}}, \mathbf{c}=\frac{\partial \mathbf{G}}{\partial \Delta \bar{\epsilon}^{\mathrm{p}}}, d=\frac{\partial \mathrm{g}}{\partial \Delta \bar{\epsilon}^{\mathrm{p}}}$.

After some mathematical developments, the above expressions can be written as:

$\mathbf{A}=\mathbf{I}_{4}+2 G \Delta \bar{e}^{\mathrm{p}} \mathbf{Q}_{\mathrm{F}}, \quad \mathbf{c}=\mathbf{V}_{\mathrm{F}}$,

$\mathbf{b}=\mathbf{V}_{\mathrm{F}}, \quad d=-\boldsymbol{H}_{\mathrm{F}}^{\prime}$,

where

$\mathbf{V}_{\mathrm{F}}=\frac{\partial \bar{\sigma}}{\partial \boldsymbol{\sigma}_{\mathrm{F}}^{\prime}}, \mathbf{Q}_{\mathrm{F}}=\frac{\partial^{2} \bar{\sigma}}{\partial \sigma_{\mathrm{F}}^{\prime 2}}$ and $H_{\mathrm{F}}^{\prime}=\partial Y / \partial \bar{\varepsilon}^{\mathrm{p}}$.

The details concerning the previously mentioned analytical derivatives are presented in Appendix A for the CPB06 yield criterion, as implemented in DD3IMP [22]. The tensor $\mathbf{Q}$ corresponds to the second order derivative of the yield criterion with respect to the effective stress state $\boldsymbol{\Sigma}=\boldsymbol{\sigma}^{\prime}$, and it is used both in the state update algorithm and on the definition of the consistent elastoplastic modulus,

$\mathbf{C}^{\mathrm{ep}}=\mathbf{C}^{\mathrm{e}}-4 G^{2}(1-\beta)\left(\frac{\mathbf{V}_{\mathrm{F}} \otimes \mathbf{V}_{\mathrm{F}}}{H_{\mathrm{F}}^{\prime}}+\Delta \overline{\mathrm{e}}^{\mathrm{p}} \mathbf{Q}_{\mathrm{F}}\right) \boldsymbol{\Lambda}$.

$\boldsymbol{\Lambda}$ depends on the hardening law adopted, given in general terms as

$\boldsymbol{\Lambda}^{-1}=\left[\mathbf{I}_{4}+2 \mu\left(\frac{\mathbf{V}_{\mathrm{F}} \otimes \mathbf{V}_{\mathrm{F}}}{H_{\mathrm{F}}^{\prime}}+\Delta \overline{\mathrm{\epsilon}}^{\mathrm{p}} \mathbf{Q}_{\mathrm{F}}\right)\right]$,

where $\mathbf{I}_{4}$ corresponds to the forth order unit symmetric tensor, defined as $I_{i j k l}=1 / 2\left(\delta_{i k} \delta_{j l}+\delta_{i l} \delta_{j k}\right)$. Since the strain increment can be either elastic or plastic, parameter $\beta$ and $(1-\beta)$ is introduced in Eq. (26) to separate, respectively, the elastic and elastoplastic parts of the total strain increment. Further details about the calculus of the parameter $\beta$ are given in Appendix A.

\subsection{Cazacu, Plunkett and Barlat yield criterion}

In order to describe both anisotropy and tension-compression asymmetry, Cazacu, Plunkett and Barlat proposed a macroscopic elastoplastic yield criterion for textured metals. The equivalent stress $\bar{\sigma}$ associated with the orthotropic form of the CPB06 yield criterion is given as

$\bar{\sigma}=B\left[\left(\left|s_{1}\right|-k s_{1}\right)^{a}+\left(\left|s_{2}\right|-k s_{2}\right)^{a}+\left(\left|s_{3}\right|-k s_{3}\right)^{a}\right]^{\frac{1}{a}}$,

where $a$ and $k$ are material parameters and $s_{1}, s_{2}, s_{3}$ are the principal values of $\mathbf{s}=\mathbf{C} \boldsymbol{\sigma}^{\prime}$, determined following Barlat et al. [27] (see Appendix A for further details). $\sigma^{\prime}$ is the deviatoric stress tensor and $\mathbf{C}$ is the constant 4th-order linear transformation tensor, given by
$\mathbf{C}=\left[\begin{array}{llllll}C_{11} & C_{12} & C_{13} & 0 & 0 & 0 \\ C_{12} & C_{22} & C_{23} & 0 & 0 & 0 \\ C_{13} & C_{23} & C_{33} & 0 & 0 & 0 \\ 0 & 0 & 0 & C_{44} & 0 & 0 \\ 0 & 0 & 0 & 0 & C_{55} & 0 \\ 0 & 0 & 0 & 0 & 0 & C_{66}\end{array}\right]$.

For a 3-D stress state, $\mathbf{C}$ involves 9 independent anisotropy coefficients. $a$ is a positive integer usually taken as 2 . As taken from Cazacu et al. the only restrictions imposed on the tensor $\mathbf{C}$ are that it satisfies the major and minor symmetries and to be invariant with respect to the orthotropy group [6]. Considering isotropic conditions, the coefficient $k$ alone allows for the description of the SD effect, giving a direct measure of the ratio between tensile and compressive yield stresses, as

$k=\frac{1-h}{1+h}$, With $h=\left[\frac{2^{a}-2\left(\frac{\sigma^{\mathrm{T}}}{\sigma^{\mathrm{C}}}\right)^{a}}{\left(2 \frac{\sigma^{\mathrm{T}}}{\sigma^{\mathrm{C}}}\right)^{a}-2}\right]^{1 / a}$.

This means that the $\sigma^{\mathrm{T}} / \sigma^{\mathrm{C}}$ ratio, i.e. the ratio between the yield stress in uniaxial tension and the yield stress in uniaxial compression, for the three principal axes is the same, in isotropic conditions. However, for an anisotropic material it is not possible to define a single $\sigma^{\mathrm{T}} / \sigma^{\mathrm{C}}$ ratio and, consequently, the physical significance of the $k$ parameter is lost [28]. $B$ is a constant defined such that $\bar{\sigma}$ reduces to the tensile yield stress along the rolling direction, and is simply defined as

$B=\left[\frac{1}{\left(\left|\phi_{1}\right|-k \phi_{1}\right)^{a}+\left(\left|\phi_{2}\right|-k \phi_{2}\right)^{a}+\left(\left|\phi_{3}\right|-k \phi_{3}\right)^{a}}\right]^{\frac{1}{a}}$,

with

$\left\{\begin{array}{l}\phi_{1} \\ \phi_{2} \\ \phi_{3}\end{array}\right\}=\left\{\begin{array}{l}(2 / 3) C_{11}-(1 / 3) C_{12}-(1 / 3) C_{13} \\ (2 / 3) C_{21}-(1 / 3) C_{22}-(1 / 3) C_{23} \\ (2 / 3) C_{31}-(1 / 3) C_{32}-(1 / 3) C_{33}\end{array}\right\}$.

For $a=2, k=0$ and $\mathbf{C}$ equal to the 4th-order identity tensor, the von Mises yield criterion is recovered. Convexity is guaranteed for any integer $a \geq 2$ and $k \in[-1,1][1]$.

\section{Application to zirconium and AZ31-Mg alloy}

The CPB06 yield criterion is used in this section to describe the anisotropic behavior of two metallic materials, known for exhibiting strong tension-compression asymmetry: Zirconium [6] and the AZ31-Mg alloy [16]. These materials were selected since the anisotropy parameters for the CPB06 yield criterion were previously determined $[6,16]$.

The CB2001 yield criterion is also used to evaluate the impact in the numerical results of neglecting the strength differential effect, although taking into account the orthotropic behavior. In this context, a four-point beam bending test is used in order to analyze both the strain components distributions in the beam central cross-section, as well the punch force evolution. This test was selected because experimental results are available for the Zirconium [6].

In brief, the CB2001 yield criterion is a generalization of the Drucker's isotropic criterion to orthotropy [3] and, in its general form, is given by 
$\bar{\sigma}=\left\{27\left[\left(J_{2}^{0}\right)^{3}-c\left(J_{3}^{0}\right)^{2}\right]\right\}^{\frac{1}{6}}$,

where $J_{2}^{0}$ and $J_{3}^{0}$ are the second and third generalized invariants of the effective stress tensor $\boldsymbol{\Sigma}$, defined as

$$
\begin{aligned}
J_{2}^{0}= & \frac{a_{1}}{6}\left(\Sigma_{11}-\Sigma_{22}\right)^{2}+\frac{a_{2}}{6}\left(\Sigma_{11}-\Sigma_{33}\right)^{2}+\frac{a_{3}}{6}\left(\Sigma_{11}-\Sigma_{33}\right)^{2}, \\
& +a_{4} \Sigma_{12}^{2}+a_{5} \Sigma_{13}^{2}+a_{6} \Sigma_{23}^{2}
\end{aligned}
$$

$$
\begin{aligned}
J_{3}^{0}= & (1 / 27)\left(b_{1}+b_{2}\right) \Sigma_{11}^{3}+(1 / 27)\left(b_{3}+b_{4}\right) \Sigma_{22}^{3} \\
& +(1 / 27)\left[2\left(b_{1}+b_{4}\right)-b_{2}-b_{3}\right] \Sigma_{33}^{3} \\
& -(1 / 9)\left(b_{1} \Sigma_{22}+b_{2} \Sigma_{33}\right) \Sigma_{11}^{2}-(1 / 9)\left(b_{3} \Sigma_{33}+b_{4} \Sigma_{11}\right) \Sigma_{22}^{2} \\
& -(1 / 9)\left[\left(b_{1}-b_{2}+b_{4}\right) \Sigma_{11}+\left(b_{1}-b_{3}+b_{4}\right) \Sigma_{22}\right] \Sigma_{33}^{2} \\
& +(2 / 9)\left(b_{1}+b_{4}\right) \Sigma_{11} \Sigma_{22} \Sigma_{33} \\
& -\left(\Sigma_{13}^{2} / 3\right)\left[2 b_{9} \Sigma_{22}-b_{8} \Sigma_{33}-\left(2 b_{9}-b_{8}\right) \Sigma_{11}\right] \\
& -\left(\Sigma_{12}^{2} / 3\right)\left[2 b_{10} \Sigma_{33}-b_{5} \Sigma_{22}-\left(2 b_{10}-b_{5}\right) \Sigma_{11}\right] \\
& -\left(\Sigma_{23}^{2} / 3\right)\left[\left(b_{6}-b_{7}\right) \Sigma_{11}-b_{6} \Sigma_{22}-b_{7} \Sigma_{33}\right]+2 b_{11} \Sigma_{12} \Sigma_{23} \Sigma_{13},
\end{aligned}
$$

where $c, a_{1}, \ldots, a_{6}$ and $b_{1}, \ldots, b_{11}$ are the anisotropy parameters. $\Sigma_{i j}, i, j=1,2,3$ are the effective stress components defined in the material frame. When considering metal sheets, the off-plane parameters cannot be experimentally evaluated. Thus, $a_{5}, a_{6}$ and $b_{k}$ $(k=6,7,8,9,11)$ are assumed as equal to the isotropic values, i.e. 1.0. For further details about the implementation and parameters identification procedure please refer to [29].

\subsection{Parameters identification for the zirconium and AZ31Mg alloy}

For the Zirconium and the AZ31-Mg alloy under study, the anisotropy parameters of the CPB06 yield criterion were previously identified in [6] and [16], respectively. In those studies, different sets of anisotropy parameters were identified for different levels of the plastic work. However, in order to keep the focus of the present work, such evolution of the anisotropy is not taken into account. Based on [6] and [16], the Zirconium presents the higher strength differential effect for a plastic strain value of $15 \%$, while the AZ31-Mg shows higher strength differential effect for about $3 \%$ of plastic strain. Thus, the anisotropy parameters selected from [6] and [16] are the ones corresponding to these values of plastic strain, in order to highlight the strength differential effect. These anisotropy parameters were used to estimate virtual experimental data for both materials. The use of this virtual data, instead of the experimental one, guarantees that the material is perfectly described by the CPB06 yield criterion. Therefore, the parameters identified using the current implementation of the CPB06 yield criterion, should accurately describe the generated virtual data. Moreover, since the off-plane parameters cannot be experimentally evaluated, in this work a new set of constitutive CPB06 parameters was identified, for each material, imposing the corresponding isotropic values, i.e. $C_{44}=C_{55}=1.0$.

Based on the sets of parameters from [6] and [16], the virtual data generated for the previously mentioned values of plastic strain is presented in Tables 1 and 2, for the Zirconium and the AZ31-Mg alloy, respectively. The virtual data includes uniaxial tensile and compression tests, equibiaxial tension and disc compression tests. Note that under this conditions it is assumed that both materials' plastic behavior is perfectly described by the CPB2006 yield criterion.

A set of anisotropy parameters, for each yield criteria, was then determined using the DD3MAT in-house code [2], taking into account the abovementioned virtual set of experimental data and $a=2$. The anisotropy coefficients should be determined such that
Table 1

Virtual data for the Zirconium, reproduced considering the anisotropy parameters of the СРВ06 yield criterion given in [6] for $15 \%$ of equivalent plastic strain.

\begin{tabular}{lllll}
\hline Test direction & $r$-value (tension) & $r$-value (compression) & $\sigma_{\theta}^{\mathrm{T}}[\mathrm{MPa}]$ & $\sigma_{\theta}^{\mathrm{C}}[\mathrm{MPa}]$ \\
\hline $0^{\circ}$ & 6.825 & -26.292 & 175.00 & 199.37 \\
$15^{\circ}$ & 7.627 & -24.961 & 164.03 & 184.45 \\
$30^{\circ}$ & 9.191 & -23.612 & 147.65 & 161.55 \\
$45^{\circ}$ & 10.126 & -22.421 & 142.12 & 152.19 \\
$60^{\circ}$ & 9.761 & -20.500 & 150.81 & 159.17 \\
$75^{\circ}$ & 8.510 & -18.278 & 171.05 & 179.12 \\
$90^{\circ}$ & 7.773 & -17.220 & 184.45 & 192.70 \\
$\sigma_{b}^{\mathrm{T}}[\mathrm{MPa}]$ & 550.87 & & & \\
$r_{b}$ & 0.532 & & & \\
\hline
\end{tabular}

Table 2

Virtual data for the AZ31-Mg alloy, reproduced considering the anisotropy parameters of the CPB06 yield criterion given in [16] for $3 \%$ of equivalent plastic strain.

\begin{tabular}{lllll}
\hline Test direction & $r$-value (tension) & $r$-value (compression) & $\sigma_{\theta}^{\mathrm{T}}[\mathrm{MPa}]$ & $\sigma_{\theta}^{C}[\mathrm{MPa}]$ \\
\hline $0^{\circ}$ & 1.082 & -0.304 & 157.12 & 92.68 \\
$15^{\circ}$ & 0.913 & -0.337 & 167.68 & 95.17 \\
$30^{\circ}$ & 0.541 & -0.403 & 198.95 & 101.30 \\
$45^{\circ}$ & 0.364 & -0.445 & 226.10 & 107.16 \\
$60^{\circ}$ & 0.792 & -0.445 & 202.00 & 109.07 \\
$75^{\circ}$ & 1.386 & -0.406 & 169.15 & 107.50 \\
$90^{\circ}$ & 1.681 & -0.384 & 157.74 & 106.32 \\
$\sigma_{b}^{\mathrm{T}}[\mathrm{MPa}]$ & 213.31 & & & \\
$r_{b}$ & 0.579 & & & \\
\hline
\end{tabular}

the yield criterion reproduces the materials' mechanical behavior as close as possible. In DD3MAT, the identification procedure is based on the minimization of an error function, using a downhill simplex method, which evaluates and weights the differences between the values determined from the constitutive equations and the experimental ones, as follow

$$
\begin{aligned}
F(\mathbf{A})= & \sum_{\theta=0}^{90} w_{\sigma_{\theta}^{\mathrm{T}}}\left(\sigma_{\theta}^{\mathrm{T}}(\mathbf{A}) / \sigma_{\theta}^{\mathrm{T}}-1\right)^{2}+\sum_{\theta=0}^{90} w_{\sigma_{\theta} \mathrm{C}}\left(\sigma_{\theta}^{\mathrm{C}}(\mathbf{A}) / \sigma_{\theta}^{\mathrm{C}}-1\right)^{2} \\
& +\sum_{\theta=0}^{90} w_{r_{\theta}}\left(r_{\theta}(\mathbf{A}) / r_{\theta}-1\right)^{2}+w_{\sigma_{b}}\left(\sigma_{b}^{\mathrm{T}}(\mathbf{A}) / \sigma_{b}^{\mathrm{T}}-1\right)^{2} \\
& +w_{r_{b}}\left(r_{b}(\mathbf{A}) / r_{b}-1\right)^{2},
\end{aligned}
$$

where A represents the set of parameters associated with the selected yield criterion. $\sigma_{\theta}^{\mathrm{T}}, \sigma_{\theta}^{\mathrm{C}}$ and $r_{\theta}$ are the experimental yield stresses in tension, compression and $r$-values determined in uniaxial tension, respectively, obtained from the uniaxial tests for a specific orientation $(\theta)$ with respect to the rolling direction (RD). $\sigma_{b}^{\mathrm{T}}$ is the experimental yield stress obtained from the equibiaxial tensile test, $r_{b}$ the experimental $r$-value obtained from the disc compression test. $\sigma_{\theta}^{\mathrm{T}}(\mathbf{A}), \sigma_{\theta}^{\mathrm{C}}(\mathbf{A}) r_{\theta}(\mathbf{A}), \sigma_{b}^{\mathrm{T}}(\mathbf{A})$ and $r_{b}(\mathbf{A})$ are the correspondent values predicted from the adopted yield criterion. Such procedure can be considered a generalization of the one proposed by Banabic et al. [30], where the weighting factors, $w_{\sigma_{\theta}^{\mathrm{T}}}$, $w_{\sigma_{\theta}}, w_{r_{\theta}}, w_{\sigma_{b}}$ and $w_{r_{b}}$ are used to balance the influence of the experimental data. Nevertheless, the selection of the weighting factors is normally a manual procedure, strongly dependent on users' expertise and knowledge. In this work, all the weighting factors are considered equal to 1.0 .

The yield stress values presented in Tables 1 and 2 correspond to the initial yield surface, such that the yield stress $Y_{0}$ is always equal to the one obtained for the uniaxial tensile test performed along the $\mathrm{RD}, \sigma_{\mathrm{RD}}^{\mathrm{T}}$. Previous results indicate that, for the $\mathrm{CPB06}$, the value proposed for the yield stress, $Y_{0}$, defined by the hardening 
Table 3

Literature and identified parameters for the Zirconium for both yield criteria. $\left(C_{44}=C_{55}=1.0\right.$ and $a_{5}, a_{6}$ and $b_{k}$ with $(k=6,7,8,9,11)$ also considered 1.0$)$.

\begin{tabular}{|c|c|c|c|c|c|c|c|c|}
\hline СРВ06 [6] & $\begin{array}{l}G_{11} \\
\mathbf{1 . 0 0 0 0}\end{array}$ & $\begin{array}{l}C_{22} \\
\mathbf{0 . 7 2 0 1}\end{array}$ & $\begin{array}{l}C_{33} \\
\mathbf{1 . 1 8 0 6}\end{array}$ & $\begin{array}{l}C_{66} \\
\mathbf{2 . 9 1 9 5}\end{array}$ & $\begin{array}{l}C_{23} \\
\mathbf{1 . 5 2 1 2}\end{array}$ & $\begin{array}{l}C_{13} \\
\mathbf{1 . 6 3 5 3}\end{array}$ & $\begin{array}{l}C_{12} \\
\mathbf{3 . 1 3 5 1}\end{array}$ & $\begin{array}{l}k \\
-0.1828\end{array}$ \\
\hline СРB06 & $\begin{array}{l}C_{11} \\
-1.1201\end{array}$ & $\begin{array}{l}C_{22} \\
-0.9378\end{array}$ & $\begin{array}{l}C_{33} \\
0.0712\end{array}$ & $\begin{array}{l}C_{66} \\
-1.7037\end{array}$ & $\begin{array}{l}C_{23} \\
-0.0347\end{array}$ & $\begin{array}{l}G_{13} \\
-0.1507\end{array}$ & $\begin{array}{l}C_{12} \\
0.2943\end{array}$ & $\begin{array}{l}k \\
0.1646\end{array}$ \\
\hline CB2001 & $\begin{array}{l}c \\
-0.6880 \\
b_{1} \\
1.3207\end{array}$ & $\begin{array}{l}a_{1} \\
1.7462 \\
b_{2} \\
1.5103\end{array}$ & $\begin{array}{l}a_{2} \\
0.1003 \\
b_{3} \\
1.4147\end{array}$ & $\begin{array}{l}a_{3} \\
0.0084 \\
b_{4} \\
0.0496\end{array}$ & $\begin{array}{l}a_{4} \\
1.926703 \\
b_{5} \\
1.2485\end{array}$ & $\begin{array}{l}b_{10} \\
1.6950\end{array}$ & & \\
\hline
\end{tabular}

Table 4

Literature and identified parameters for the AZ31-Mg for both yield criteria $\left(C_{44}=C_{55}=1.0\right.$ and $a_{5}, a_{6}$ and $b_{k}$ with $(k=6,7,8,9,11)$ also considered 1.0$)$.

\begin{tabular}{|c|c|c|c|c|c|c|c|c|}
\hline СРВ06 [16] & $\begin{array}{l}C_{11} \\
\mathbf{1 . 0 0 0 0}\end{array}$ & $\begin{array}{l}C_{22} \\
\mathbf{1 . 0 9 0 0}\end{array}$ & $\begin{array}{l}C_{33} \\
\mathbf{3 . 3 4 2 0}\end{array}$ & $\begin{array}{l}C_{66} \\
\mathbf{0 . 7 3 0 0}\end{array}$ & $\begin{array}{l}C_{23} \\
\mathbf{0 . 2 4 3 0}\end{array}$ & $\begin{array}{l}C_{13} \\
\mathbf{0 . 0 9 8 0}\end{array}$ & $\begin{array}{l}C_{12} \\
-\mathbf{0 . 1 6 8 0}\end{array}$ & $\begin{array}{l}k \\
-0.625\end{array}$ \\
\hline СРВ06 & $\begin{array}{l}C_{11} \\
1.0526\end{array}$ & $\begin{array}{l}C_{22} \\
1.1556\end{array}$ & $\begin{array}{l}C_{33} \\
3.5000\end{array}$ & $\begin{array}{l}C_{66} \\
0.7591\end{array}$ & $\begin{array}{l}C_{23} \\
0.2527\end{array}$ & $\begin{array}{l}G_{13} \\
0.0955\end{array}$ & $\begin{array}{l}C_{12} \\
-0.1663\end{array}$ & $\begin{array}{l}k \\
-0.6344\end{array}$ \\
\hline CB2001 & $\begin{array}{l}c \\
1.1369 \\
b_{1} \\
3.0707\end{array}$ & $\begin{array}{l}a_{1} \\
1.4620 \\
b_{2} \\
-0.1938\end{array}$ & $\begin{array}{l}a_{2} \\
0.7157 \\
b_{3} \\
-0.5420\end{array}$ & $\begin{array}{l}a_{3} \\
0.7464 \\
b_{4} \\
-2.2672\end{array}$ & $\begin{array}{l}a_{4} \\
0.3996 \\
b_{5} \\
-0.3057\end{array}$ & $\begin{array}{l}b_{10} \\
0.0531\end{array}$ & & \\
\hline
\end{tabular}

law, should be in accordance with the $\sigma_{\mathrm{RD}}^{\mathrm{T}}$ to enable an accurate description of the in-plane yield stresses [28]. For both yield criteria the minimization problem associated to Eq. (33) is over constrained, since that a total of 23 experimental values are used to identify only 8 parameters in case of CPB06, and 11 parameters in case of CB2001. Considering that the problem is over constrained, the $r$-values in uniaxial compression are not used, to check if it is possible to captured them using the remaining data, for a material that follows the CPB06 yield criterion. A summary of the sets of parameters determined for both yield criteria is presented in Tables 3 and 4 . The yield criterion parameters used to generate the virtual experimental data are also depicted (first line).

Figs. 1 and 2 and present the comparison between numerical and experimental $r$-values and normalized yield stresses in tension and compression for both yield criteria and both materials, respectively. The figures also present the $r$-values determined in uniaxial compression, although not used in the identification procedure. Note that for the CB2001 the $r$-values and yield stress in-plane evolution in tension and compression is the same. Regarding the Zirconium, the $r$-values are accurately described by both yield criteria with negligible differences between them. For the AZ31-Mg alloy the same accurate description is observed.

For the Zirconium, the yield stresses are accurately predicted with the СРB06 yield criterion, while the CB2001 presents a less accurate fit with the increase of the angle relative to the rolling direction. As for the AZ31-Mg, both yield criteria show an accurate description of the yield stresses directionalities.

The yield surfaces predicted with both yield criteria are presented in Figs. 3 and 4, for the plane $\sigma_{1}, \sigma_{2}$ (with $\sigma_{3}=0$ ) and the $\pi$-plane, for the Zirconium and the AZ31-Mg alloy, respectively. The rolling (RD), transverse (TD) and normal (ND) directions correspond to 1,2 and 3, respectively. Note that the equibiaxial stress state is equivalent to the through-thickness uniaxial compression, i.e. there is an equivalence between the stress ratio in ND and equibiaxial $\left(\sigma_{3}^{\mathrm{T}} / \sigma_{3}^{\mathrm{C}}\right)=\left(\sigma_{b}^{\mathrm{C}} / \sigma_{b}^{\mathrm{T}}\right)$ [28], as shown in the figures.

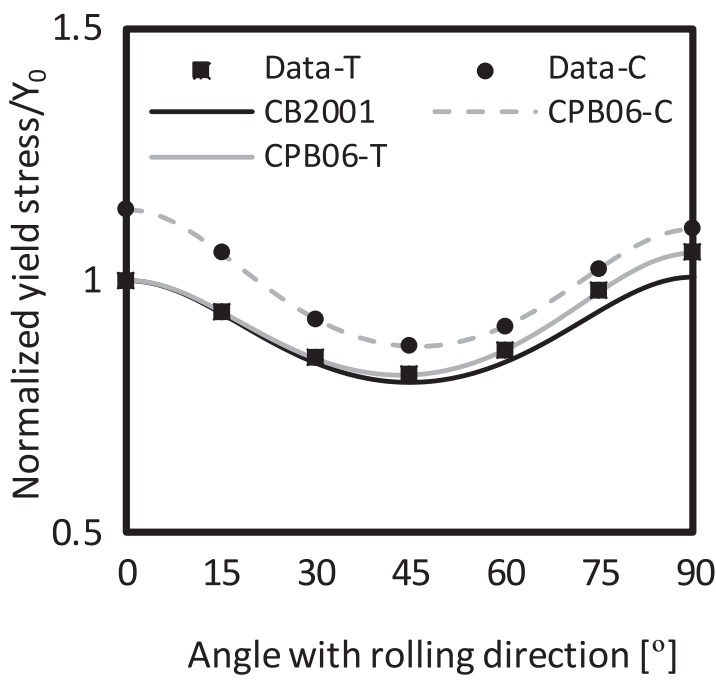

(b)

(a)

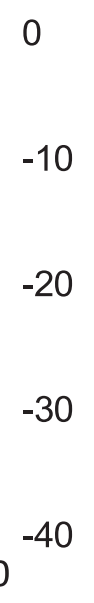

Angle with rolling direction $\left[{ }^{\circ}\right]$

Fig. 1. Virtual and predicted (a) $r$-values and (b) normalized yield stresses for the Zirconium. 


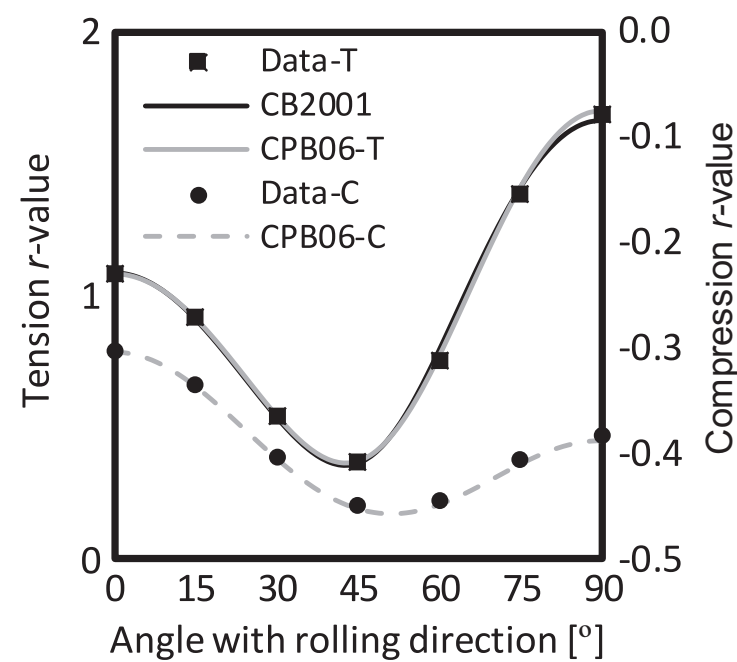

(a)

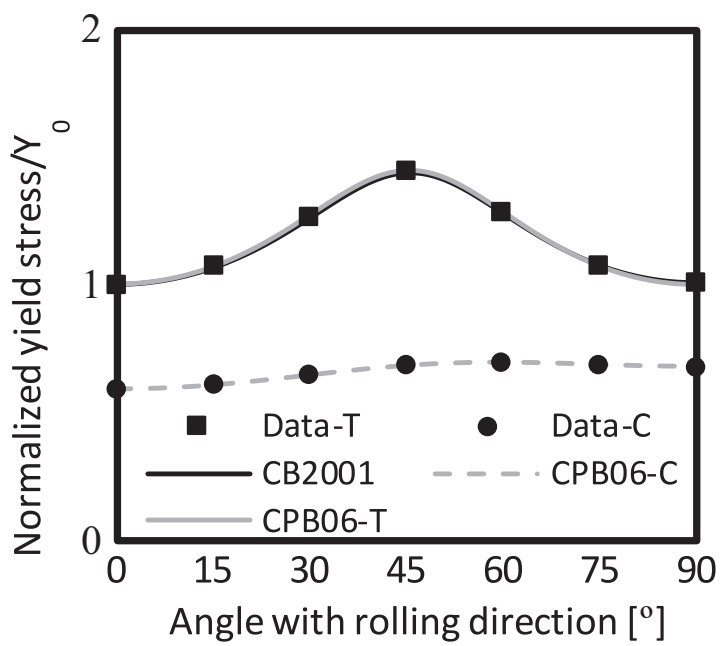

(b)

Fig. 2. Virtual and predicted (a) $r$-values and (b) normalized yield stresses for the AZ31-Mg alloy.

Fig. 3 shows that the yield surface for the Zirconium is well described by the CPB06 yield criterion for all stress states. Note the fact that there is a slight difference between numerical and virtual data for the point relative to equibiaxial compression, $\sigma_{b}^{\mathrm{C}}$. Moreover, as expected, the CB2001 cannot predict the tension-compression asymmetry, falling short mainly in the point relative to equibiaxial compression, $\sigma_{b}^{\mathrm{C}}$.

Fig. 4 shows that both the CРB06 and CB2001 accurately predict the material behavior in the first quadrant of the plane $\sigma_{1}, \sigma_{2}$, where tension-compression asymmetry has no influence. However, in the other quadrants, the CB2001 cannot accurately predict the material behavior, due to the point-symmetry of the criterion, while the CРB06 reproduces closely the experimental material behavior. Although CPB06 has a reduced set of anisotropy parameters, both yield criteria are flexible enough to enable the accurate description of the in-plane anisotropy of both $r$-values and flow stresses (yield stresses).

Comparing the CPB06 anisotropy parameters proposed in the literature with the ones identified with DD3MAT (see Table 3) for Zirconium, it should be mentioned that the parameter $k$ changes from negative to positive, without affecting the predicted tensioncompression ratios obtained for the orthotropy axes (see Table 5), highlighting that both sets of parameters are robust. Although not shown here, it should be mentioned that it is also possible to obtain a set of anisotropy parameters for the CРB06 yield criterion with a negative value for the parameter $k$. In that case the anisotropy parameters become symmetrical to the ones presented in Table 3, for the CPB06 yield criterion.

For the AZ31-Mg alloy, the obtained anisotropy parameters are very similar to the ones proposed on the literature (see Table 4), leading to similar stress ratios for the orthotropy axes (see Table 6). In this case, it is not possible to obtain a set of parameters with a positive value for $k$ that accurately describes the material behavior, which seems to result from the "triangular" shape with rounded corners of the yield surface, in opposition to the elliptical shape of the Zirconium yield locus.

Regarding the tension-compression ratios for the orthotropy axes, for the Zirconium (see Table 5), the values obtained for the $\sigma_{1}$ and $\sigma_{2}$ directions are relatively close and lower than 1.0.

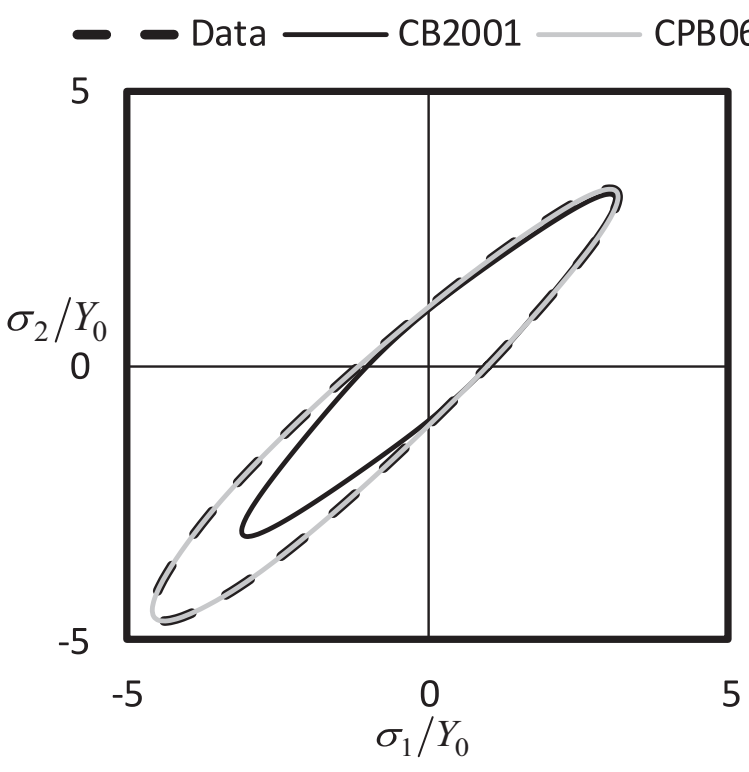

(a)
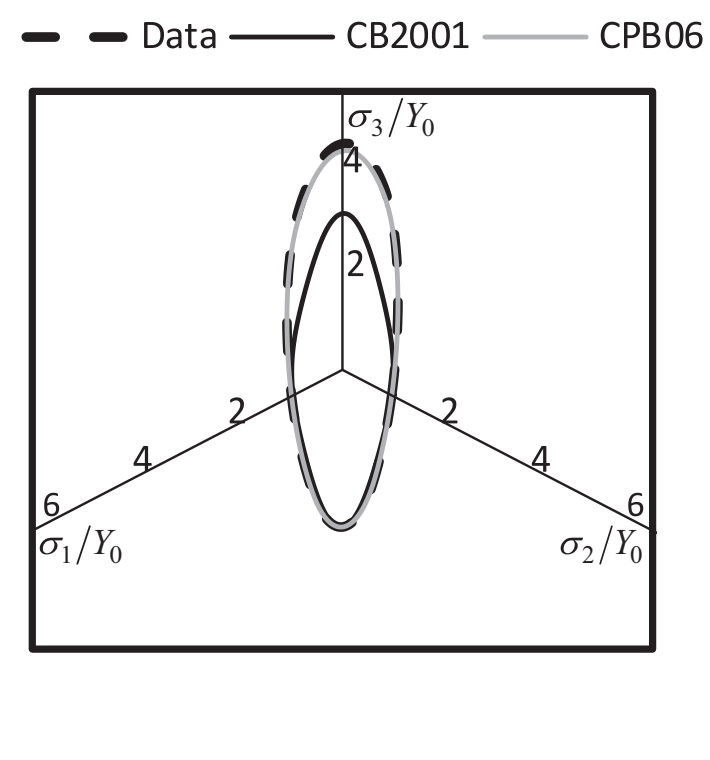

(b)

Fig. 3. Predicted yield surfaces in the (a) $\sigma_{1} / Y_{0}, \sigma_{2} / Y_{0}$ plane and (b) $\pi$-plane for the Zirconium. 


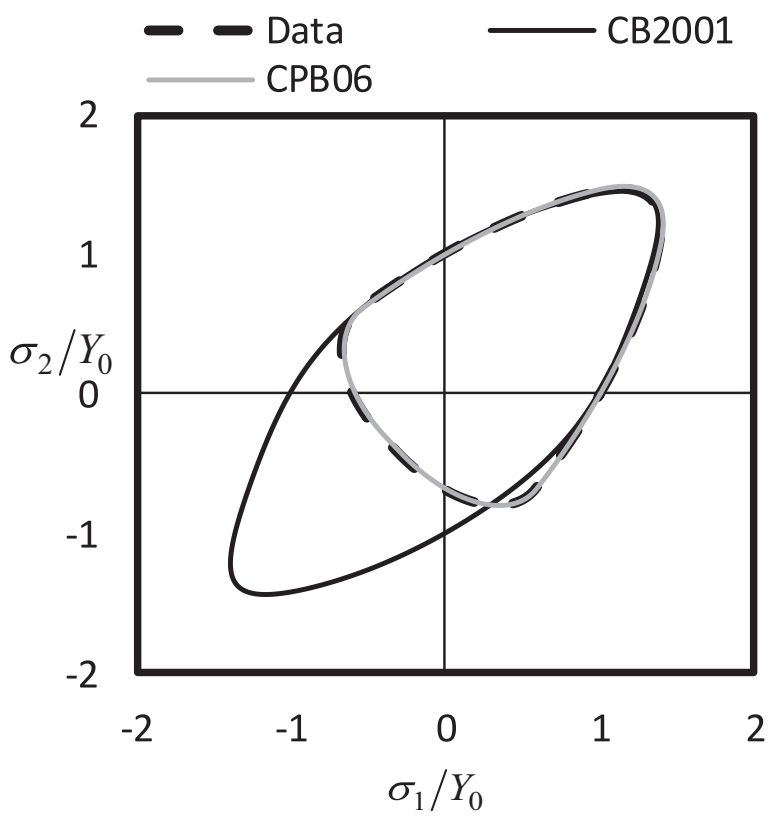

(a)

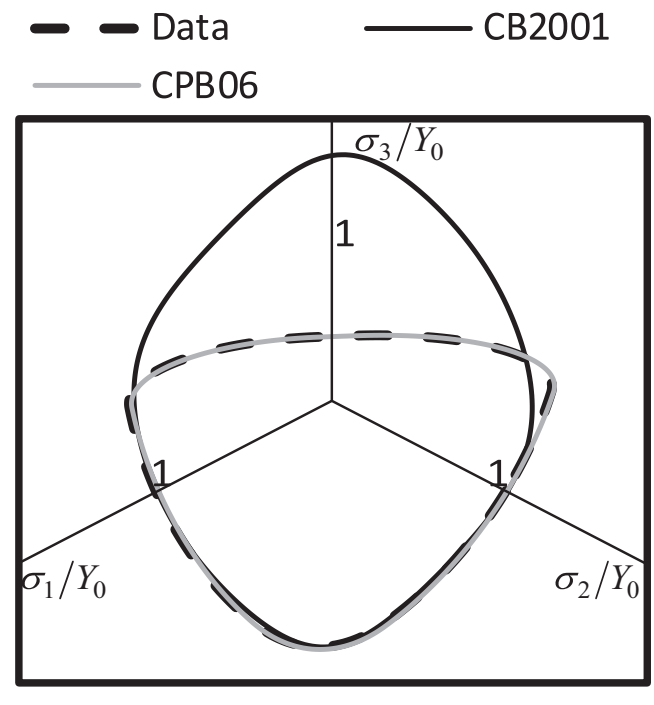

(b)

Fig. 4. Predicted yield surfaces in the (a) $\sigma_{1} / Y_{0}, \sigma_{2} / Y_{0}$ plane and (b) $\pi$-plane for the AZ31-Mg alloy.

Table 5

Ratios obtained for the three principal axes for the Zirconium.

\begin{tabular}{llll}
\hline & $\left(\sigma_{1}^{\mathrm{T}} / \sigma_{1}^{\mathrm{C}}\right)$ & $\left(\sigma_{2}^{\mathrm{T}} / \sigma_{2}^{\mathrm{C}}\right)$ & $\left(\sigma_{3}^{\mathrm{T}} / \sigma_{3}^{\mathrm{C}}\right)$ \\
\hline Experimental & 0.8778 & 0.9572 & 1.4474 \\
CPB06 & 0.8767 & 0.9569 & 1.3940 \\
\hline
\end{tabular}

Table 6

Ratios obtained for the three principal axes for the AZ31-Mg alloy.

\begin{tabular}{llll}
\hline & $\left(\sigma_{1}^{\mathrm{T}} / \sigma_{1}^{\mathrm{C}}\right)$ & $\left(\sigma_{2}^{\mathrm{T}} / \sigma_{2}^{\mathrm{C}}\right)$ & $\left(\sigma_{3}^{\mathrm{T}} / \sigma_{3}^{\mathrm{C}}\right)$ \\
\hline Experimental & 1.6953 & 1.4837 & 0.2607 \\
CPB06 & 1.6998 & 1.4715 & 0.2573 \\
\hline
\end{tabular}

Considering the $\sigma_{3}$ direction, the ratio is higher than 1.0 with a slight difference between numerical and experimental values, as supported by Fig. 3(b). Regarding the AZ31-Mg alloy (see Table 6), the ratios obtained for the $\sigma_{1}$ and $\sigma_{2}$ directions are relatively close and higher than 1.0, meaning that the yield stresses in tension are higher than in compression. On the other hand, the ratio in $\sigma_{3}$ is of about 0.26 , meaning that the yield stress in compression is four times higher than the yield stress in tension. Note that, as shown in Fig. 4(b), the ratio in $\sigma_{3}$ is the inverse of the ratio in the equibiaxial direction.

\subsection{Four-point bending test}

The beam bending example considered is based on the original work of Kaschner [31]. The simulation of the four-point bending

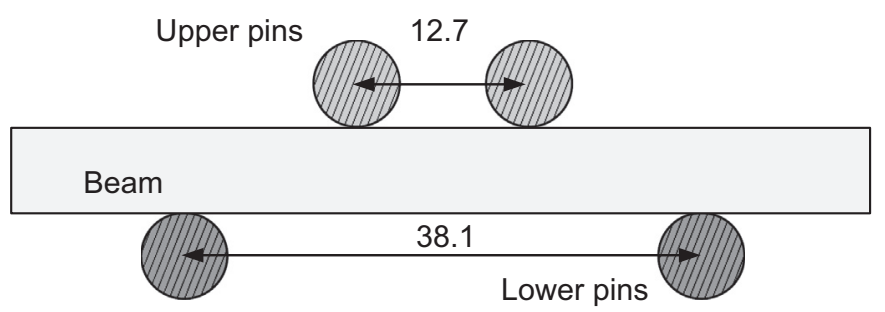

(a)

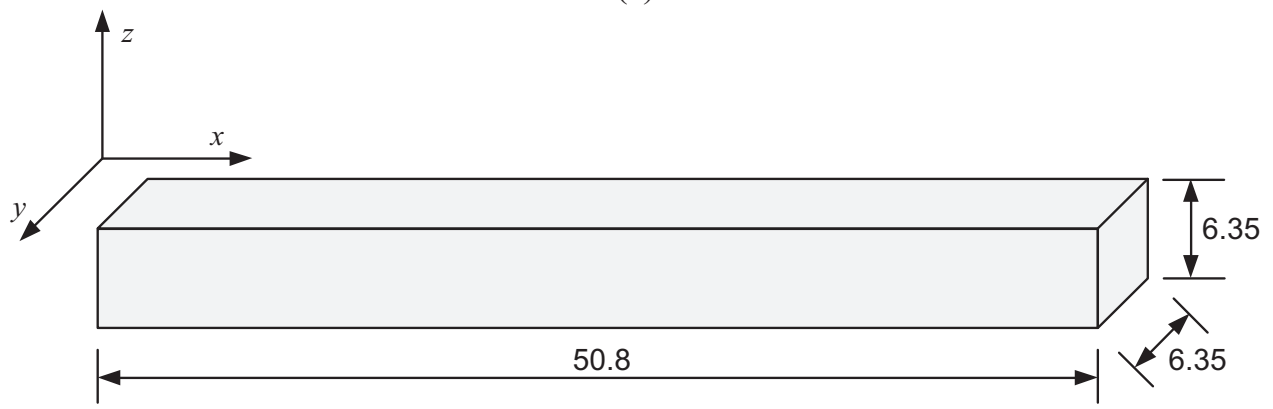

(b)

Fig. 5. Schematic of the (a) four-point bending test and (b) beam geometry and dimensions (dimensions in mm). 


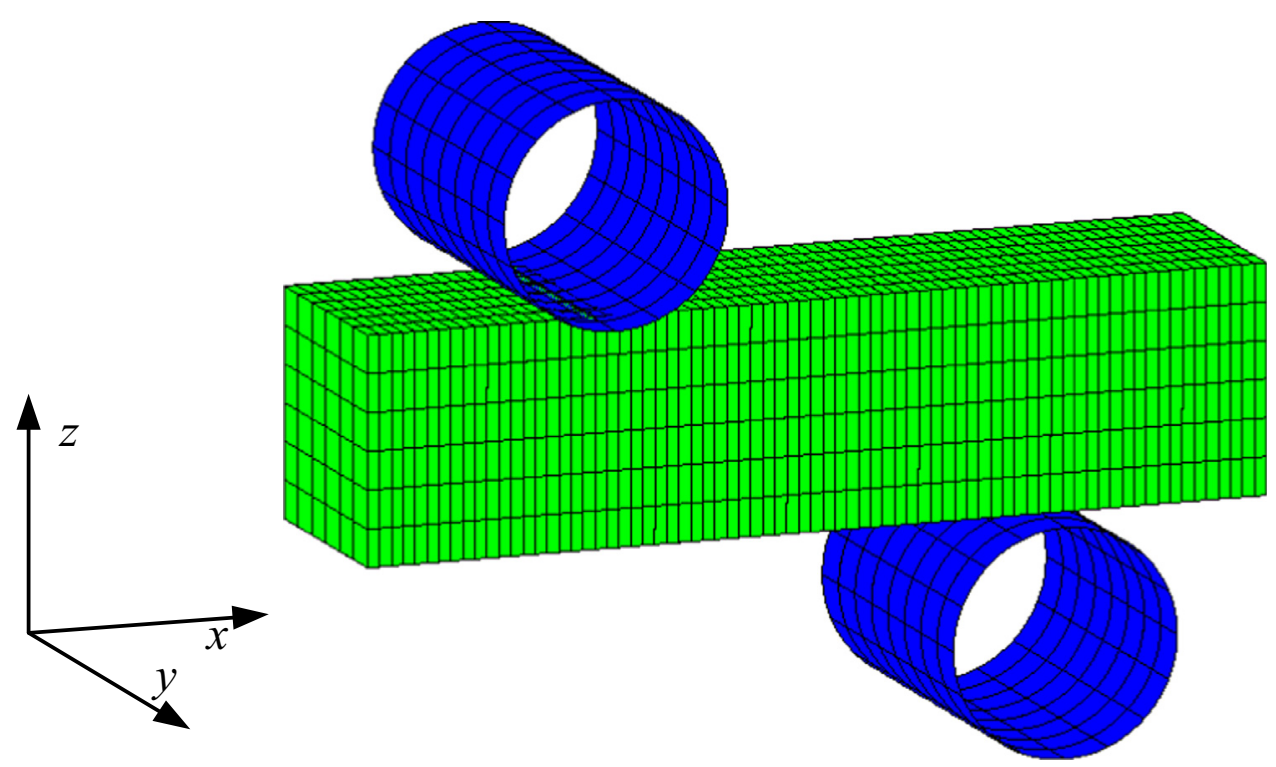

Fig. 6. Numerical model of the four-point bending test (half model).

tests is performed with both the CPB06 and CB2001 yield criteria. This test is used in order to highlight the strain distribution and shift of the neutral layer in materials which exhibit tensioncompression asymmetry, and the importance of accurately modeling this phenomenon. The four-point bending fixture is composed by two upper pin dowels, having a downwards displacement of $6 \mathrm{~mm}$, and two lower pins which are held stopped. The center distance between the upper pins is $12.7 \mathrm{~mm}$ and for the lower pins is $38.1 \mathrm{~mm}$ (Fig. 5). Fig. 6 presents the numerical model of the four-point bending test. The dimensions of the beam are $6.35 \mathrm{~mm} \times 6.35 \mathrm{~mm} \times 50.8 \mathrm{~mm}$.

The beam is discretized with 8 -node hexahedral finite elements, combined with a selective reduced integration technique [32]. Due to geometrical and material symmetries, only half of the global structure is modeled with the beam composed by $6 \times 6$ elements and 75 elements along the beam axes, yielding a total of 2700 finite elements.

For each material, two different beam orientations are considered, both taken with the RD aligned with the $x$-axis: (i) case TD, the TD is coincident with the global $y$-axis, i.e. the material axes are coincident with the global ones; and (ii) case ND, the ND is aligned with the global $y$, i.e. the beam is rotated $90^{\circ}$ around the $x$-axis. This rotation allows the change in orientation of the hard/ soft to deform direction relatively to the load direction. In this work, an isotropic work hardening law is considered, assuming that the initial shape of the yield surface expands only isotropically with plastic deformation.

\section{Results and discussion}

Fig. 7(a) presents a comparison of the predicted normalized punch force evolution obtained for Zirconium, with both yield criteria, showing that the differences observed with the change of orientation are negligible. Nonetheless, the force predicted by the СРB06 is higher, when compared with the CB2001. This can be related to the fact that yielding in compression is lower for the CB2001.

Fig. 7(b) presents the same comparison regarding the AZ31-Mg alloy. When using the CB2001 yield criterion, the difference between orientations are, again, negligible. When using the СРВ06, however, the differences are evident, with the TD case presenting

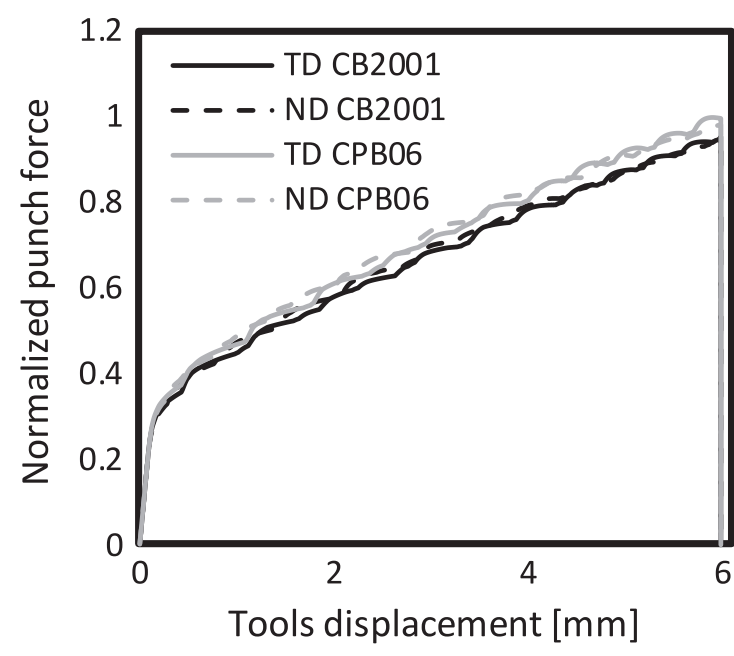

(a)

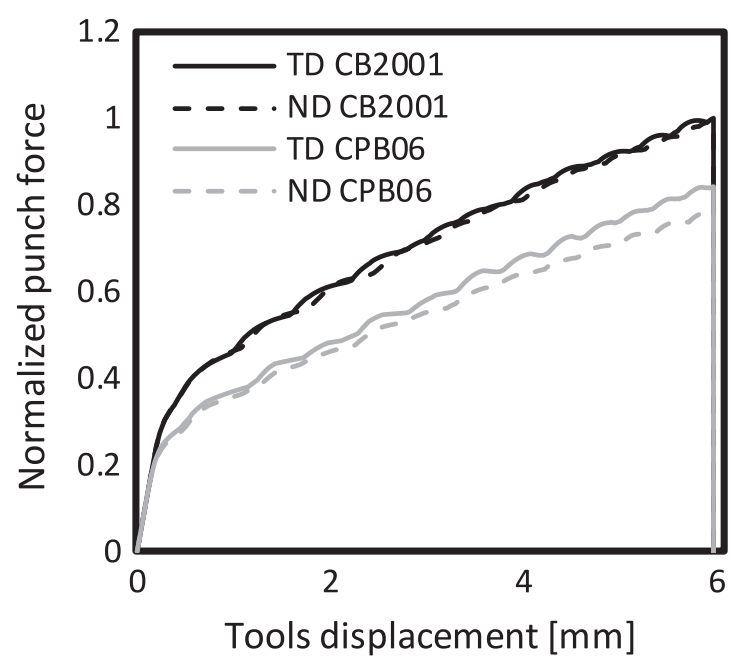

(b)

Fig. 7. Predicted punch force evolution for both CPB06 and CB2001 yield criteria, for (a) Zirconium and (b) AZ31-Mg alloy. 
higher values than the ND case. For this material, the CB2001 predicts higher yield stresses in compression when compared with the CPB06, thus resulting in a higher punch force evolution for CB2001.

Regarding the predicted deformed bar central cross-section, the results for the Zirconium are presented in Fig. 8, for both orientations. Note that for the Zirconium, the experimentally measured strain distributions are available, as well as the $y-z$ cross-sections of the deformed bars [6]. Considering the TD case, the final shapes obtained for both yield criteria are in good agreement with the experimental ones, with negligible differences between both. As for the ND case, the final shape is very close to the initial one, for both yield criteria. This means that both yield criteria correctly model the rigidity of the bar in the hard to deform $\langle c\rangle$-axis direction. This is coherent with the fact that the Zirconium presents a strong basal structure, i.e. $\langle c\rangle$-axis of the crystals predominantly oriented along the plate normal direction. Hence, in the TD case, the $\langle c\rangle$-axis, are mostly aligned with the $z$-axis while for the ND case, the $\langle c\rangle$-axis are mostly aligned with the $y$-axis. In fact, even though the CB2001 does not allow describing the compression yield stresses evolution, that evolution is very close in shape to the experimental one, with only slightly lower values (see Fig. 1(b)). Moreover, as shown in Fig. 1(a) the in-plane $r$-values present high absolute values in order to describe the lack of ability to deform of the ND direction. The fact that the CРB06 presents negative uniaxial compression $r$-values does not seems to affect the final geometry of the beam central cross-section.

Fig. 9 shows the deformed bars central cross-section for the AZ31-Mg alloy. For this material, when considering the TD case, the CB2001 yield criterion predicts a wedge shape, similar to the one obtained for the Zirconium. The CPB06, predicts a barrel-like shape. In fact, for the beam section bellow the neutral layer, the shape predicted for both yield criteria is very similar. Above that, the CB2001 predicted section dilates in the $y$ direction, with the $z$ one almost constant. For the СРB06, the section narrows in the $y$ direction and dilates in the $z$ direction. Also note that the curvature on the top surface has different directions for both yield criteria. Regarding the ND case, the CB2001 is not sensitive to the change in orientation. For the СРВ06, however, the shape becomes closer to the one obtained with the CB2001 yield criterion but with more pronounced features, mainly in the upper part of the beam. For both cases, note that the major differences appear in the layer which is subjected to compression states, for which the CPB06 reproduces the virtual compression yield stresses in-plane evolution. The CB2001 presents a compression yield stresses inplane evolution that is neither similar in shape or values to the virtual, i.e. shows a more anisotropic behavior with overestimated values (see Fig. 2(b)). Moreover, as shown in Fig. 2(a), the СРВ06 predicts negative in-plane uniaxial compression $r$-values. In particular the $r_{0}$ is close to the one in tension, for both yield criteria, while it is close to -0.304 for the compression predicted by the CPB06. These may explain the fact that the deformation in the ND direction is always higher than the one along TD direction for the layers submitted to compression when using the CРB06 yield criterion. Also, it can explain why, in the TD case, the beam increases height while it narrows in the $y$ direction, i.e, the strains in both direction are symmetric, being coherent with the fact that the $r$-value is negative.

In order to better understand the differences between both yield criteria and material orientations, the strain distributions are compared, along the $z$-axis of the central cross-section (for $y=0$, according to Figs. 8 and 9). Again, experimental data is available only for the Zirconium, obtained through digitally processed local strain measurements determined by a dot-matrix deposition and mapping (DMDM) technique [31]. Fig. 10 presents the comparison between experimental and numerically predicted Green-Lagrange strain components, for the Zirconium. The Green-Lagrange strain tensor is defined by $\mathbf{E}=1 / 2(\mathbf{C}-\mathbf{I})=1 / 2\left(\mathbf{F F}^{\mathrm{T}}-\mathbf{I}\right)$, where $\mathbf{F}$ is the deformation gradient. Regarding the TD case (see Fig. 10(a)), both yield criteria present a good prediction of the strain components distribution when compared with the experimental ones. However, the СРВ06 predicts closer results as well as the shift of the neutral layer. For the ND case, the trend is the same - both yield criteria show a good agreement with the experimental data, with the CPB06 able to have a more accurate prediction, including the shift of the neutral layer. Also, this analysis allows to confirm the consistency of results between the predicted deformed shapes presented in Fig. 8, with the predictions of both yield criteria relatively close. In fact, the negative values predicted by the СРВ06 for the uniaxial compression $r$-values only seem to affect the Green-Lagrange strain distribution for the hard to deform direction which, in the compression layer, are closer to zero when comparing with the ones obtained by the CB2001. As previously mentioned, in the TD case the hard to deform direction is mostly aligned with the $z$-axis, while for the ND case is mostly aligned with the $y$-axis. It should also be mentioned that the test leads to a strain of about $20 \%$ in the outmost fibers of the beam [6], which is close to the plastic strain value of $15 \%$ selected to determine the yield locus for the Zirconium.

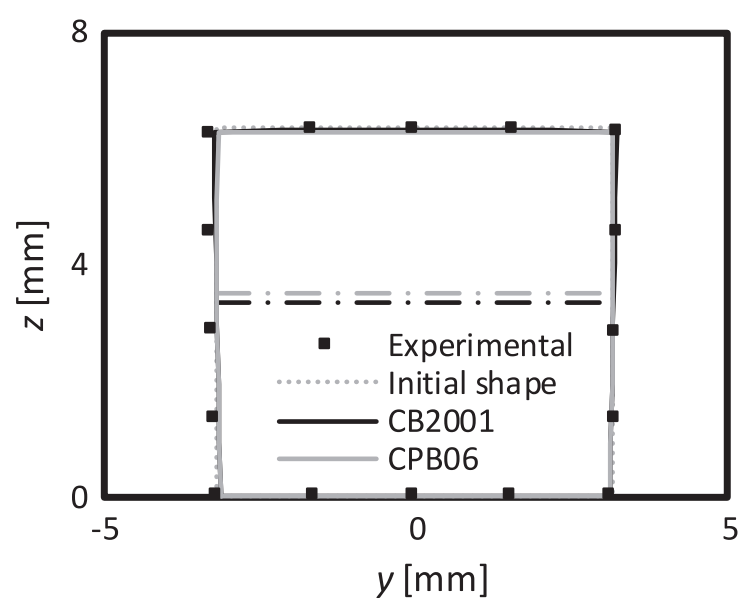

(b)

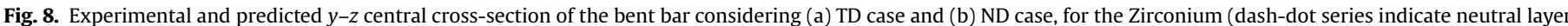
for both yield criteria). 


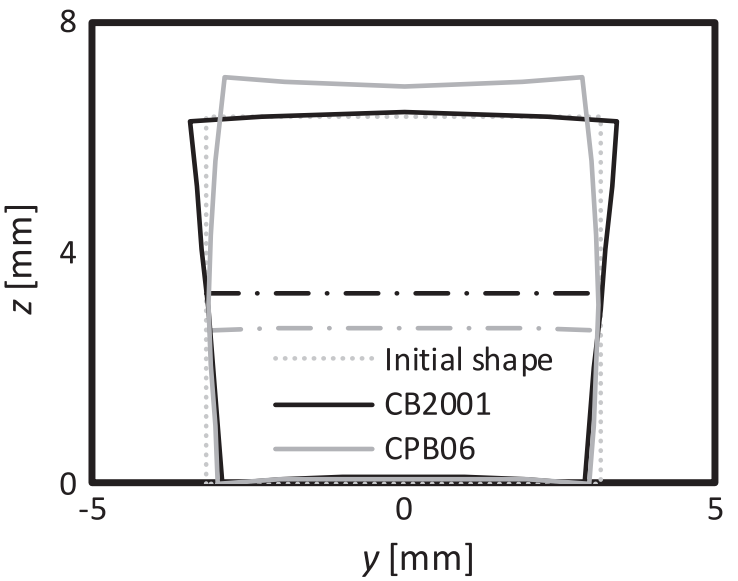

(a)

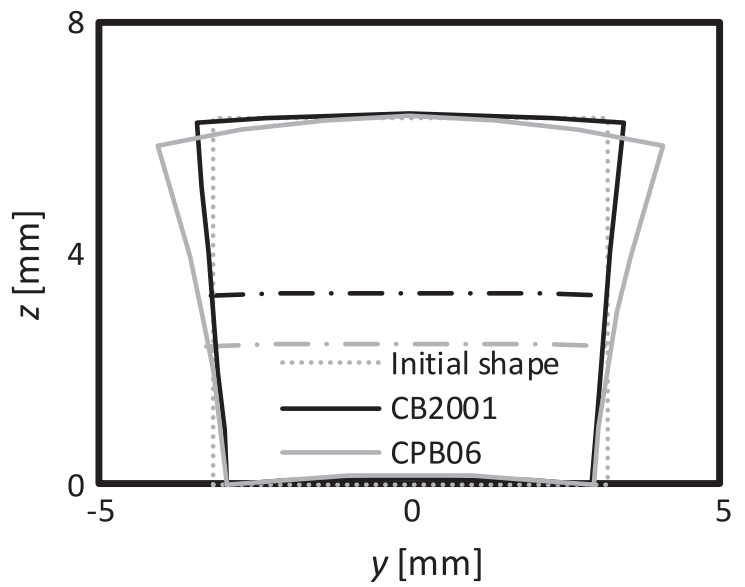

(b)

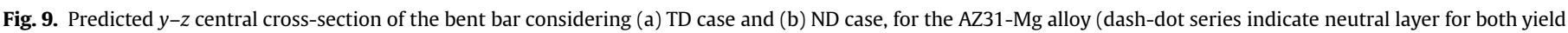
criteria).

For the AZ31-Mg alloy, the results comparing only the numerically predicted strain components obtained along the $z$-axis of the beam central cross-section are presented in Fig. 11. Regarding the TD case, the CB2001 predicts strains in agreement with the final deformed shape obtained (see Fig. 9). Also note that components $E_{z z}$ and $E_{y y}$ have a very close evolution. As for the СРВ06, there are major differences when comparing with the CB2001. The $E_{y y}$ component is always negative with an inversion in the zone relative to the neutral layer. This evolution explains the final deformed barrel-like shape (see Fig. 9). The $E_{z z}$ component starts at the beam bottom layer at about -0.7 and at the upper layer with a value of about 0.38 . This evolution is in agreement with the fact that the beam central cross-section suffers a considerable increase in height. For the ND case, $E_{z z}$ is almost constant and negative, thus explaining the minor change in height of the central cross-section. The $E_{y y}$ component, however, shows a variation similar with the $E_{z z}$ one for the TD case. This evolution is responsible for the more pronounced wedge-like shape, mainly for the area above the neutral layer. Nonetheless, it is not expected that the shape of the

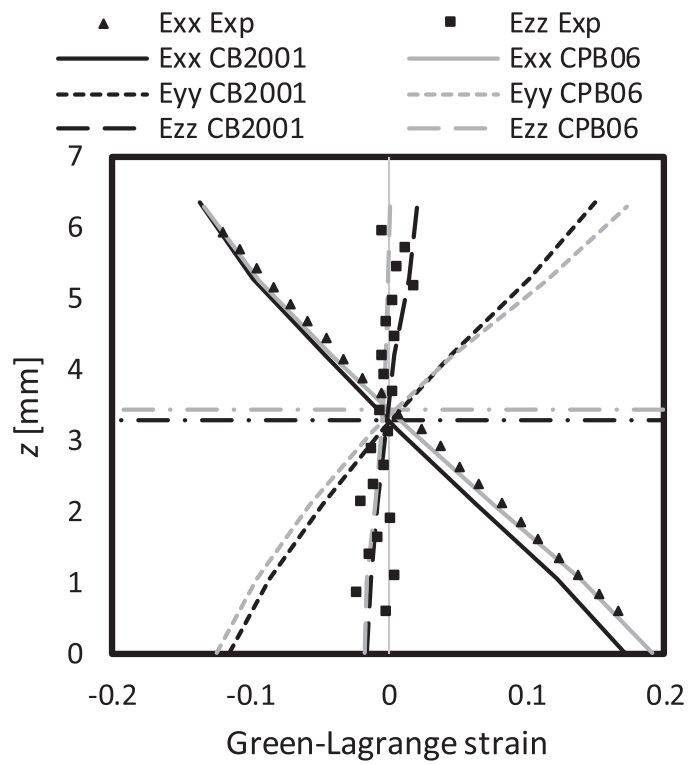

(a) beam for the case of the AZ31-Mg alloy is so pronounced, since the tension-compression ratio decreases with the increase of plastic deformation (see [16]). In this case, the strain value attained in the outmost fibers of the beam is quite distinct from the plastic strain value of $3 \%$ selected to determine the yield locus.

\section{Conclusion}

The main features of the implementation of the Cazacu, Plunkett and Barlat yield criterion in the implicit in-house finite element solver DD3IMP were described. The analytical expressions for the first and second derivatives of the CPB06 yield criterion, necessary for an improved solution of the stress update algorithm, were derived and shown in detail in Appendix A, enabling its implementation in other finite element codes. The anisotropy parameters for Zirconium and AZ31-Mg alloy, both known for exhibiting strong tension-compression asymmetry as well as inplane anisotropy, were identified for both CPB06 and CB2001 yield

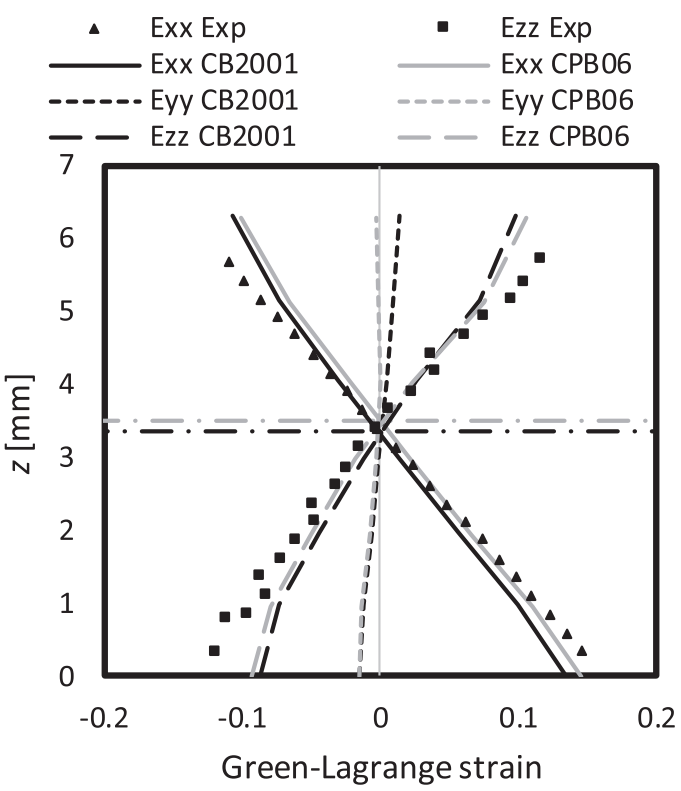

(b)

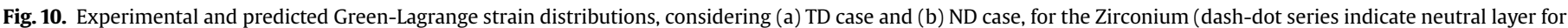
both yield criteria). 


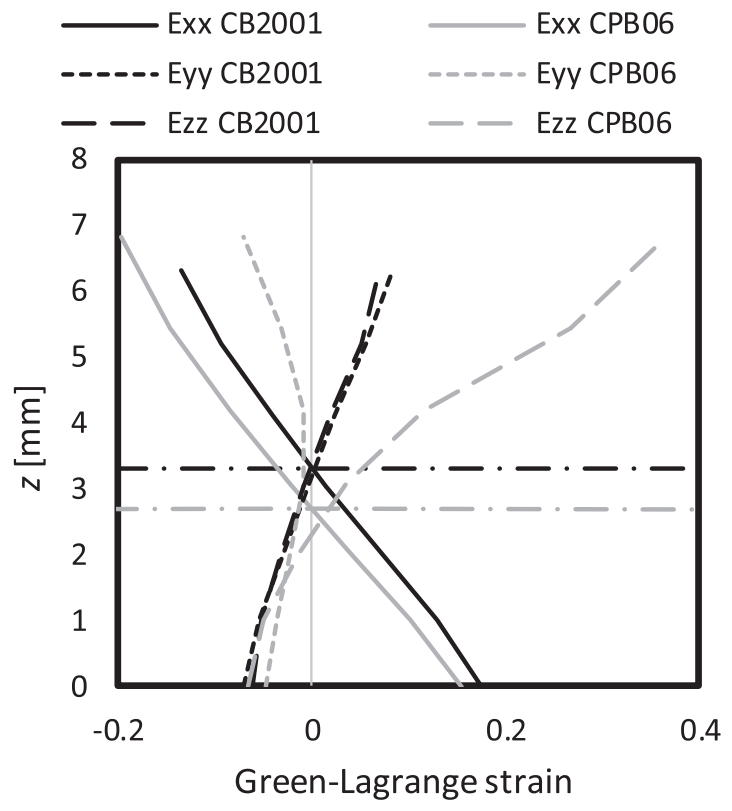

(a)

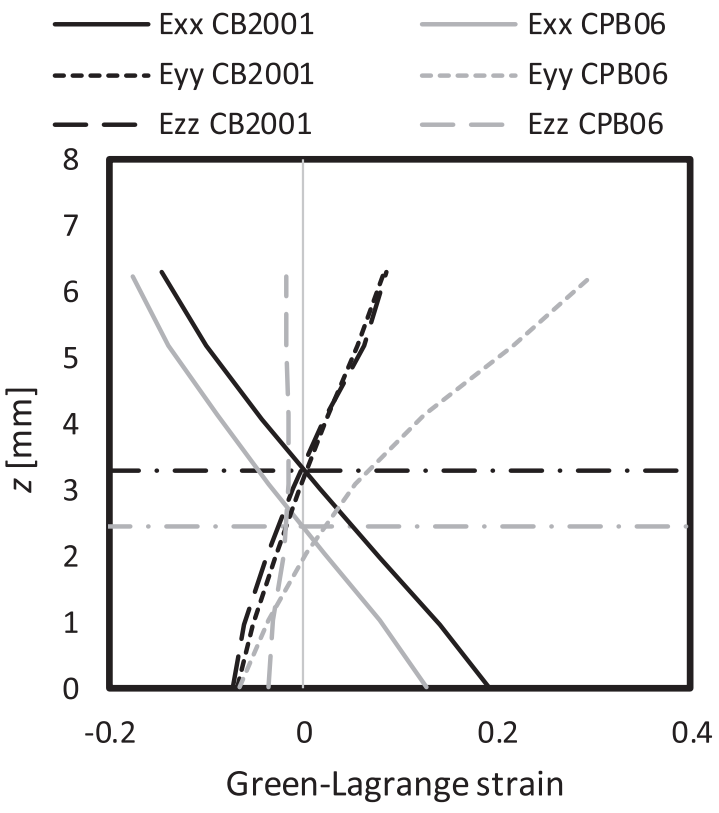

(b)

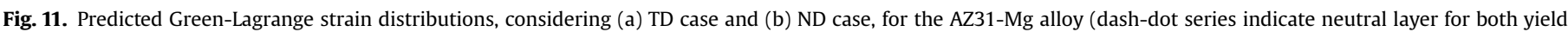
criteria).

criteria. The results show that the yield criteria adopted can accurately describe both the yield stresses and $r$-values for tensile stress states, with CPB06 yield criterion also allowing an accurately prediction of the mechanical behavior for compression stress states, for both materials.

The numerical simulation of a four-point bending test was performed, considering the beam aligned along two different orientations. The results show that an accurate description of the tension-compression asymmetry influences the punch force evolution, for both materials. This effect is more relevant for the AZ31$\mathrm{Mg}$ which shows a higher tension-compression asymmetry. In what concerns Zirconium and regarding the shape of the central cross-sections, both yield criteria describe well the final deformed shape with minimal differences between them, for both beam orientations, meaning that the tension-compression asymmetry is not the predominant phenomenon contributing to the almost squared shape for the ND case. In fact, the strain distribution analysis shows that both yield criteria have a good approximation to the experimental values, with the CРB06 showing better accuracy, particularly in what concerns the prediction of the shift of the neutral layer.

For the AZ31-Mg alloy and analyzing the deformed shape for the TD orientation, the difference between both yield criteria is clearly visible. The strain distributions predicted by the CPB06 show a very different trend from the CB2001, converging on a strong shift of the neutral layer. When considering the ND orientation, the CB2001 shows negligible differences in both shape and strain distributions on the central cross-section, when compared with the ones obtained for the TD case. In the ND case, the CPB06 predicts a shape similar to the one predicted by the CB2001, but more pronounced, supported by the strain distribution predicted. Thus, highlighting the sensitivity to the orientation of the СРВ06 yield criterion. In summary, the global results shown and discussed, put in evidence the importance of modeling and taking into account the tension-compression asymmetry when analyzing the deformation of HCP materials.

\section{Acknowledgment}

The authors gratefully acknowledge the financial support of the Portuguese Foundation for Science and Technology (FCT), Portugal via the projects PTDC/EME-TME/118420/2010, UID/EMS/00285/ 2013 and by FEDER, Portugal funds through the program COMPETE - Programa Operacional Factores de Competitividade, under the project CENTRO-07-0224-FEDER-002001 (MT4MOBI). The first author is also grateful to the FCT for the Ph.D. Grant SFRH/BD/ $98545 / 2013$.

\section{Appendix A. Analytical expressions of the derivatives of the CPB2006 yield criterion}

A.1. This section presents the details concerning the calculation of the following quantities

$\beta, \mathbf{V}=\frac{\partial \bar{\sigma}}{\partial \boldsymbol{\sigma}}, \mathbf{Q}=\frac{\partial^{2} \bar{\sigma}}{\partial \boldsymbol{\sigma}^{2}}$,

specifically related with the numerical implementation of the CPB06 yield criterion in the FE solver DD3IMP.

The scalar $\beta$ has to be evaluated whenever the effective stress state at the beginning of the time increment is elastic. It is determined with the consistency condition, such that

$\Phi(\beta)=\bar{\sigma}\left(\boldsymbol{\sigma}_{0}^{\prime}, \Delta \boldsymbol{\epsilon}^{\prime}, \beta\right)-Y_{I}=0$,

where subscript $I$ denotes the flow stress at the beginning of the time increment. $\beta$ is the only unknown of this non-linear equation. Thus, the Newton-Raphson method is used to iteratively determine $\beta$, given by the relation

$\beta^{i+1}=\beta^{i}-\frac{\Phi^{i}}{\Phi^{i^{\prime}}}$.

A schematic representation of the stress update algorithm is shown in Fig. 12, where $\sigma^{\prime \text { trial }}$ corresponds to the one obtained from Eq. (17), and subscripts 0 and F denote the deviatoric stress tensor at the beginning and at the end of the time increment 


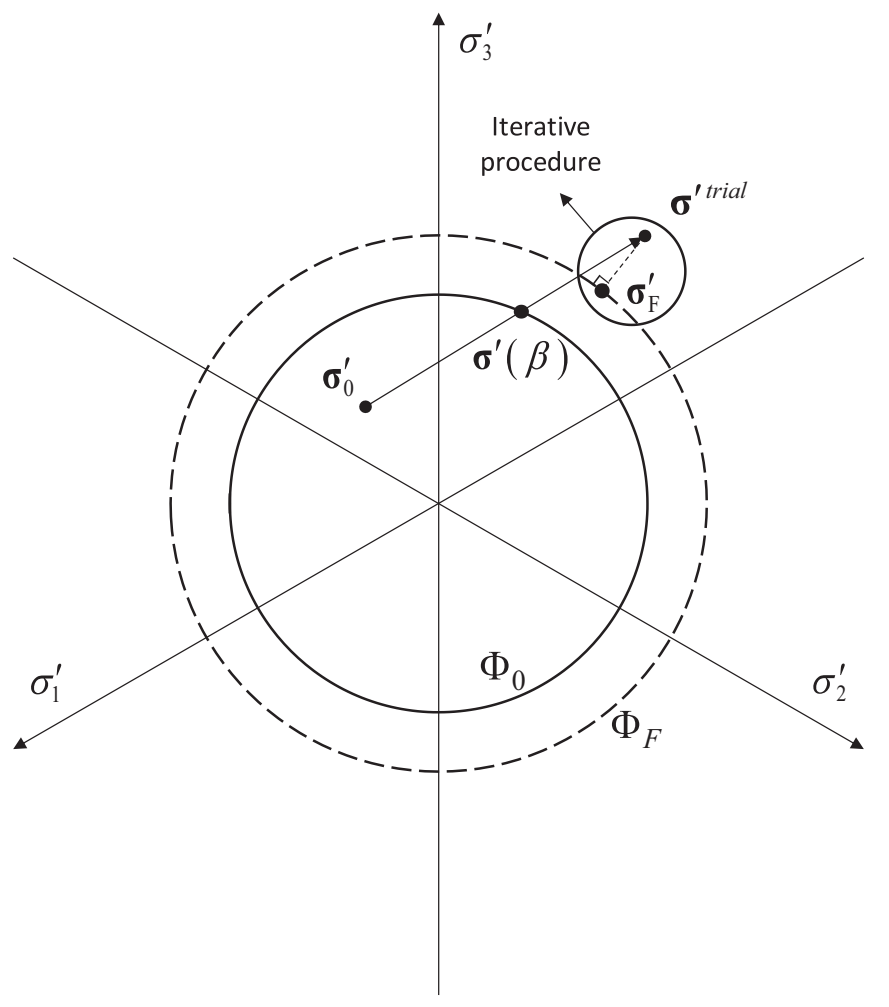

Fig. 12. Schematic representation of the stress update algorithm, with subscripts 0 and $\mathrm{F}$ denoting the quantities at the beginning and at the end of the time increment $[t, t+\Delta t]$.

\section{$[t, t+\Delta t]$, respectively.}

The scalar quantities $\Phi^{i}$ and $\Phi^{i^{\prime}}$ are required, being the first one given by Eq. (38) and the second one by

$\Phi^{i^{\prime}}=\frac{\partial \bar{\sigma}\left(\boldsymbol{\sigma}_{0}^{\prime}, \Delta \boldsymbol{\epsilon}^{\prime}, \beta\right)}{\partial \beta^{i}}=\frac{\partial \bar{\sigma}}{\partial \boldsymbol{\sigma}^{i}} \frac{\partial \boldsymbol{\sigma}^{i}}{\partial \beta^{i}}=\mathbf{V}^{i}:\left(2 \mu \Delta \mathbf{\epsilon}^{\prime}\right)$.

The tensor $\mathbf{V}$ is given by

$\mathbf{V}_{i j}=\frac{1}{2}\left(\frac{\partial}{\partial \boldsymbol{\sigma}_{i j}}+\frac{\partial}{\partial \boldsymbol{\sigma}_{j i}}\right) F=\left.\left.\frac{\partial \bar{\sigma}}{\partial s_{p}} \frac{\partial s_{p}}{\partial H_{m}} \frac{\partial H_{m}}{\partial s_{r s}}\right|^{s y m} \frac{\partial s_{r s}}{\partial \boldsymbol{\sigma}_{i j}}\right|^{s y m}$,

while the tensor $\mathbf{Q}$ is given by,

$$
\begin{aligned}
\mathbf{Q}_{i j k l}= & \frac{1}{2}\left(\frac{\partial}{\partial \boldsymbol{\sigma}_{k l}}+\frac{\partial}{\partial \boldsymbol{\sigma}_{l k}}\right) \mathbf{V}_{i j} \\
= & \frac{1}{2}\left(\frac{\partial}{\partial \boldsymbol{\sigma}_{k l}}+\frac{\partial}{\partial \boldsymbol{\sigma}_{l k}}\right)\left(\left.\left.\frac{\partial \bar{\sigma}}{\partial s_{p}} \frac{\partial s_{p}}{\partial H_{m}} \frac{\partial H_{m}}{\partial s_{r s}}\right|^{s y m} \frac{\partial s_{r s}}{\partial \boldsymbol{\sigma}_{i j}}\right|^{s y m}\right) \\
= & \frac{\partial^{2} \bar{\sigma}}{\partial s_{p} \partial s_{q}}\left(\left.\left.\frac{\partial s_{q}}{\partial H_{n}} \frac{\partial H_{n}}{\partial s_{u v}}\right|^{s y m} \frac{\partial s_{u v}}{\partial \boldsymbol{\sigma}_{k l}}\right|^{s y m}\right)\left(\left.\left.\frac{\partial s_{p}}{\partial H_{m}} \frac{\partial H_{m}}{\partial s_{r s}}\right|^{s y m} \frac{\partial s_{r s}}{\partial \boldsymbol{\sigma}_{i j}}\right|^{s y m}\right) \\
& +\frac{\partial \bar{\sigma}}{\partial s_{p}} \frac{\partial^{2} s_{p}}{\partial H_{m} \partial H_{n}}\left(\left.\left.\frac{\partial H_{n}}{\partial s_{u v}}\right|^{s y m} \frac{\partial s_{u v}}{\partial \boldsymbol{\sigma}_{k l}}\right|^{s y m}\right)\left(\left.\left.\frac{\partial H_{m}}{\partial s_{r s}}\right|^{s y m} \frac{\partial s_{r s}}{\partial \boldsymbol{\sigma}_{i j}}\right|^{s y m}\right) \\
& +\left.\left.\left.\frac{\partial \bar{\sigma}}{\partial s_{p}} \frac{\partial s_{p}}{\partial H_{m}} \frac{\partial^{2} H_{m}}{\partial s_{r s} \partial s_{u v}}\right|^{s y m} \frac{\partial s_{u v}}{\partial \boldsymbol{\sigma}_{k l}}\right|^{s y m} \frac{\partial s_{r s}}{\partial \boldsymbol{\sigma}_{i j}}\right|^{s y m}
\end{aligned}
$$

The initial effective stress state is then updated to the initial yield surface as

$$
\left(\boldsymbol{\sigma}_{0}^{\prime}\right)^{*} \leftarrow \boldsymbol{\sigma}_{0}^{\prime}+\beta\left(2 \mu \Delta \mathbf{\epsilon}^{\prime}\right)
$$

The terms relative to the first and second order derivatives are defined bellow.

\section{A.2. First derivatives}

a) Expressions for $\partial \bar{\sigma} / \partial s_{p}$

$$
\begin{aligned}
& \frac{\partial \bar{\sigma}}{\partial s_{p}}=\frac{\partial}{\partial s_{p}}\left[B \cdot\left(\left(\left|s_{1}\right|-k s_{1}\right)^{a}+\left(\left|s_{2}\right|-k s_{2}\right)^{a}+\left(\left|s_{3}\right|-k s_{3}\right)^{a}\right)\right] \\
& =\frac{B}{a}\left[\left(\left|s_{1}\right|-k s_{1}\right)^{a}+\left(\left|s_{2}\right|-k s_{2}\right)^{a}+\left(\left|s_{3}\right|-k s_{3}\right)^{a}\right]^{\frac{1}{a}-1} . \\
& \quad\left[a\left(\left|s_{p}\right|-k s_{p}\right)^{a-1}\left(\frac{\left|s_{p}\right|}{s_{p}}-k\right)\right],=R \cdot D\left(s_{p}\right)
\end{aligned}
$$

with

$R=\frac{B}{a}(A)^{\frac{1}{a}-1}$,

and

$A=\left[\left(\left|s_{1}\right|-k s_{1}\right)^{a}+\left(\left|s_{2}\right|-k s_{2}\right)^{a}+\left(\left|s_{3}\right|-k s_{3}\right)^{a}\right]$.

Also,

$D\left(s_{p}\right)=\left[a\left(\left|s_{p}\right|-k s_{p}\right)^{a-1}\left(\frac{\left|s_{p}\right|}{s_{p}}-k\right)\right]$

b) Expressions for $\partial s_{p} / \partial H_{m}$

$$
\begin{aligned}
& \frac{\partial s_{i}}{\partial H_{m}}= \frac{\partial}{\partial H_{m}}\left[H_{1}+2 \sqrt{H_{1}^{2}+H_{2}} \cdot \cos \left(\frac{\theta}{3}+\beta_{i}\right)\right], \\
& \frac{\partial s_{i}}{\partial H_{1}}= 1+\frac{2 H_{1}}{\sqrt{H_{1}^{2}+H_{2}}} \cdot \cos \left(\frac{\theta}{3}+\beta_{i}\right)-\frac{2}{3} \sqrt{H_{1}^{2}+H_{2}} \cdot \\
& \sin \left(\frac{\theta}{3}+\beta_{i}\right) \frac{\partial \theta}{\partial H_{1}}, \\
& \frac{\partial s_{i}}{\partial H_{2}}= \frac{2 H_{1}}{\sqrt{H_{1}^{2}+H_{2}}} \cdot \cos \left(\frac{\theta}{3}+\beta_{i}\right)-\frac{2}{3} \sqrt{H_{1}^{2}+H_{2}} . \\
& \sin \left(\frac{\theta}{3}+\beta_{i}\right) \frac{\partial \theta}{\partial H_{2}}, \\
& \frac{\partial s_{i}}{\partial H_{3}}=-\frac{2}{3} \sqrt{H_{1}^{2}+H_{2}} \cdot \sin \left(\frac{\theta}{3}+\beta_{i}\right) \frac{\partial \theta}{\partial H_{3}}, \\
& \text { with } \beta_{1}=0, \beta_{2}=\frac{4}{3} \pi \text { and } \beta_{3}=\frac{2}{3} \pi . \\
& \frac{\partial \theta}{\partial H_{1}}=-\frac{3}{2} \frac{\left(2 H_{1}^{2}+H_{2}\right) H_{p}-2 H_{q} H_{1}}{H_{p} \sqrt{H_{p}^{3}+H_{q}^{2}}} \\
& \frac{\partial \theta}{\partial H_{2}}=-\frac{3}{2} \frac{H_{1} H_{p}-2 H_{q}}{H_{p} \sqrt{H_{p}^{3}+H_{q}^{2}}} \\
& \frac{\partial \theta}{\partial H_{3}}=-\frac{H_{p}}{H_{p} \sqrt{H_{p}^{3}+H_{q}^{2}}}
\end{aligned}
$$

c) Expressions for $\partial H_{m} / \partial s_{r s}$

$$
\begin{aligned}
& \frac{\partial H_{1}}{\partial s_{k l}}=\frac{1}{2}\left(\frac{\partial}{\partial s_{k l}}+\frac{\partial}{\partial s_{l k}}\right) H_{1}=\frac{1}{3} \delta_{k l} \\
& \frac{\partial H_{2}}{\partial s_{k l}}=\frac{1}{2}\left(\frac{\partial}{\partial s_{k l}}+\frac{\partial}{\partial s_{l k}}\right) H_{1}=\frac{1}{3}\left[\begin{array}{l}
-s_{y y}-s_{z z} \\
-s_{x x}-s_{z z} \\
\frac{1}{2}\left(s_{z y}+s_{y z}\right) \\
\frac{1}{2}\left(s_{z x}+s_{x z}\right) \\
\frac{1}{2}\left(s_{y x}+s_{x y}\right)
\end{array}\right]
\end{aligned}
$$




$$
\begin{aligned}
\frac{\partial H_{3}}{\partial s_{k l}}= & \frac{1}{2}\left(\frac{\partial}{\partial s_{k l}}+\frac{\partial}{\partial s_{l k}}\right) H_{1} \\
= & {\left[\begin{array}{c}
s_{y y} s_{z z}-s_{y z} s_{z y} \\
s_{x x} s_{z z}-s_{x z} s_{z x} \\
s_{x x} s_{y y}-s_{x y} s_{y x} \\
\frac{1}{2}\left(s_{x y} s_{z x}+s_{y x} s_{x z}-s_{x x}\left(s_{y z}+s_{z y}\right)\right) \\
\frac{1}{2}\left(s_{y x} s_{z y}+s_{x y} s_{y z}-s_{y y}\left(s_{x z}+s_{z x}\right)\right) \\
\frac{1}{2}\left(s_{y z} s_{z x}+s_{z y} s_{x z}-s_{z z}\left(s_{x y}+s_{y x}\right)\right)
\end{array}\right] }
\end{aligned}
$$

d) Expression for $\partial s_{k l} / \partial \sigma_{i j}$

$$
\frac{\partial s_{k l}}{\partial \boldsymbol{\sigma}_{i j}}=\frac{\partial}{\partial \boldsymbol{\sigma}_{i j}}\left[\mathbf{L}_{i j k l}: \boldsymbol{\sigma}_{k l}\right]=\mathbf{L}_{i j k l} \frac{\partial \boldsymbol{\sigma}_{k l}}{\partial \boldsymbol{\sigma}_{i j}}=\mathbf{L}_{i j k l} \delta_{k i} \delta_{l j}
$$

\section{A.3. Second derivatives}

The second order derivatives have a role in the state variables update (see Eq. (56)) and in the definition of the consistent elastoplastic modulus.

a) Expression for $\partial^{2} \bar{\sigma} / \partial s_{p} \partial s_{q}$

$$
\frac{\partial^{2} \bar{\sigma}}{\partial s_{p} \partial s_{q}}=\frac{1}{A} \cdot\left(\frac{1}{a}-1\right) \cdot\left[\frac{\partial \bar{\sigma}}{\partial s_{p}}\right] \cdot D\left(s_{q}\right)+R \cdot \frac{\partial}{\partial s_{q}}\left[D\left(s_{p}\right)\right]
$$

with

$$
D\left(s_{q}\right)=\left[a\left(\left|s_{q}\right|-k s_{q}\right)^{a-1}\left(\frac{\left|s_{q}\right|}{s_{q}}-k\right)\right]
$$

b) Expressions for $\partial^{2} s_{p} / \partial H_{m} H_{n}$

$$
\begin{aligned}
& \frac{\partial^{2} s_{p}}{\partial H_{m} H_{n}}=\frac{\partial}{H_{n}}\left(\frac{\partial s_{p}}{\partial H_{m}}\right) \\
& \frac{\partial}{\partial H_{n}}\left(\frac{\partial s_{i}}{\partial H_{1}}\right)=\cos \left(\frac{\theta}{3}+\beta_{i}\right) \cdot \mathrm{dA} 1(n)-\frac{2}{3} \sin \left(\frac{\theta}{3}+\beta_{i}\right) \frac{\partial \theta}{\partial H_{1}} \mathrm{dA} 2(n) \\
& -\frac{1}{3} \sqrt{H_{1}^{2}+H_{2}}\left[\begin{array}{c}
\frac{2 H_{1}}{H_{1}^{2}+H_{2}} \sin \left(\frac{\theta}{3}+\beta_{i}\right) \\
+\frac{2}{3} \cdot \cos \left(\frac{\theta}{3}+\beta_{i}\right) \frac{\partial \theta}{\partial H_{1}}
\end{array}\right] \frac{\partial \theta}{\partial H_{n}} \\
& -\frac{2}{3} \sqrt{H_{1}^{2}+H_{2}} \cdot \sin \left(\frac{\theta}{3}+\beta_{i}\right) \frac{\partial^{2} \theta}{\partial H_{1} \partial H_{n}} \\
& \frac{\partial}{\partial H_{n}}\left(\frac{\partial s_{i}}{\partial H_{2}}\right)=\cos \left(\frac{\theta}{3}+\beta_{i}\right) \text {. } \\
& \mathrm{dA} 3(n)-\frac{2}{3} \sin \left(\frac{\theta}{3}+\beta_{i}\right) \frac{\partial \theta}{\partial \mathrm{H}_{2}} \mathrm{dA} 2(n) \\
& -\frac{1}{3} \sqrt{H_{1}^{2}+H_{2}}\left[\frac{1}{H_{1}^{2}+H_{2}} .\right. \\
& \left.\sin \left(\frac{\theta}{3}+\beta_{i}\right)+\frac{2}{3} \cos \left(\frac{\theta}{3}+\beta_{i}\right) \frac{\partial \theta}{\partial H_{2}}\right] \frac{\partial \theta}{\partial H_{n}} \\
& -\frac{2}{3} \sqrt{H_{1}^{2}+H_{2}} \cdot \sin \left(\frac{\theta}{3}+\beta_{i}\right) \frac{\partial^{2} \theta}{\partial H_{2} \partial H_{n}}
\end{aligned}
$$

$$
\begin{aligned}
\frac{\partial}{\partial H_{n}}\left(\frac{\partial s_{i}}{\partial H_{3}}\right)= & -\frac{2}{3} \sin \left(\frac{\theta}{3}+\beta_{i}\right) \frac{\partial \theta}{\partial H_{3}} \mathrm{dA} 2(n) \\
& -\frac{1}{3} \sqrt{H_{1}^{2}+H_{2}}\left[\frac{2}{3} \cos \left(\frac{\theta}{3}+\beta_{i}\right) \frac{\partial \theta}{\partial H_{3}}\right] \frac{\partial \theta}{\partial H_{n}} \\
& -\frac{2}{3} \sqrt{H_{1}^{2}+H_{2}} \cdot \sin \left(\frac{\theta}{3}+\beta_{i}\right) \frac{\partial^{2} \theta}{\partial H_{3} \partial H_{n}}
\end{aligned}
$$

with

$$
\begin{aligned}
& \mathrm{dA} 1(n)=\frac{\partial}{\partial H_{n}}\left(\frac{2 H_{1}}{\sqrt{H_{1}^{2}+H_{2}}}\right) \\
&=\left[\frac{2 H_{2}}{\left(\sqrt{H_{1}^{2}+H_{2}}\right)^{3}}-\frac{H_{1}}{\left(\sqrt{H_{1}^{2}+H_{2}}\right)^{3}} \quad 0\right] \\
& \mathrm{dA} 2(n)=\frac{\partial}{\partial H_{n}}\left(\sqrt{H_{1}^{2}+H_{2}}\right)=\left[\frac{H_{1}}{\sqrt{H_{1}^{2}+H_{2}}} \frac{1}{2 \sqrt{H_{1}^{2}+H_{2}}} 0\right] \\
& \mathrm{dA} 3(n)=\frac{\partial}{\partial H_{n}}\left(\frac{1}{\sqrt{H_{1}^{2}+H_{2}}}\right) \\
&=\left[\begin{array}{ll}
-\frac{H_{1}}{\left(\sqrt{H_{1}^{2}+H_{2}}\right)^{3}}-\frac{1}{2\left(\sqrt{H_{1}^{2}+H_{2}}\right)^{3}} & 0
\end{array}\right] \\
& \frac{\partial}{\partial H_{n}}\left(\cos \left(\frac{\theta}{3}+\beta_{i}\right)\right)=-\frac{1}{3} \sin \left(\frac{\theta}{3}+\beta_{i}\right) \frac{\partial \theta}{\partial H_{n}} \\
& \frac{\partial}{\partial H_{n}}\left(\sin \left(\frac{\theta}{3}+\beta_{i}\right)\right)=\frac{1}{3} \cos \left(\frac{\theta}{3}+\beta_{i}\right) \frac{\partial \theta}{\partial H_{n}} .
\end{aligned}
$$

Also,

$$
\begin{aligned}
\frac{\partial}{H_{n}}\left(\frac{\partial \theta}{\partial H_{m}}\right)= & -\frac{H_{p} \frac{\partial^{2} H_{q}}{\partial H_{m} \partial H_{n}}+\frac{\partial H_{q}}{\partial H_{m}} \frac{\partial H_{p}}{\partial H_{n}}-\frac{3}{2}\left(\frac{\partial H_{q}}{\partial H_{n}} \frac{\partial H_{p}}{\partial H_{m}}+H_{q} \frac{\partial^{2} H_{p}}{\partial H_{m} \partial H_{n}}\right)}{H_{p} \sqrt{H_{p}^{3}-H^{2} q}} \\
& +\frac{\left(H_{p} \frac{\partial H_{q}}{\partial H_{m}}-\frac{3}{2} H_{q} \frac{\partial H_{p}}{\partial H_{m}}\right)}{2\left(H_{p} \sqrt{H_{p}^{3}-H_{q}^{2}}\right)^{2} \sqrt{H_{p}^{3}-H^{2} q}}\left(\left(5 H_{p}^{3}-2 H_{q}^{2}\right) \frac{\partial H_{p}}{\partial H_{n}}\right. \\
& \left.-2 H_{p} H_{q} \frac{\partial H_{q}}{\partial H_{n}}\right)
\end{aligned}
$$

with

$$
\begin{aligned}
& \frac{\partial H_{p}}{\partial H_{m}}=\frac{\partial}{\partial H_{m}}\left(H_{1}^{2}+H_{2}\right)=\left[\begin{array}{l}
2 H_{1} \\
1 \\
0
\end{array}\right], \\
& \frac{\partial H_{q}}{\partial H_{m}}=\frac{\partial}{\partial H_{m}}\left[\frac{1}{2}\left(2 H_{1}^{3}+3 H_{1} H_{2}+2 H_{3}\right)\right]=\frac{1}{2}\left[\begin{array}{c}
6 H_{1}^{2}+3 H_{2} \\
3 H_{1} \\
2
\end{array}\right], \\
& \frac{\partial^{2} H_{p}}{\partial H_{m} \partial H_{n}}=\frac{\partial}{\partial H_{n}}\left(\left[\begin{array}{c}
2 H_{1} \\
1 \\
0
\end{array}\right]\right)=\left[\begin{array}{lll}
2 & 0 & 0 \\
0 & 0 & 0 \\
0 & 0 & 0
\end{array}\right], \\
& \frac{\partial^{2} H_{q}}{\partial H_{m} \partial H_{n}}=\frac{\partial}{\partial H_{n}}\left[\frac{1}{2}\left[\begin{array}{c}
6 H_{1}^{2}+3 H_{2} \\
3 H_{1} \\
2
\end{array}\right]\right]=\frac{1}{2}\left[\begin{array}{lll}
12 H_{1} & 0 & 0 \\
3 & 0 & 0 \\
0 & 0 & 0
\end{array}\right] .
\end{aligned}
$$

c) Expressions for $\partial^{2} H_{m} / \partial s_{r s} \partial s_{u v}$

$$
\frac{\partial^{2} H_{1}}{\partial s_{k l} \partial s_{m n}}=\frac{1}{2}\left(\frac{\partial}{\partial s_{m n}}+\frac{\partial}{\partial s_{n m}}\right)\left[\frac{1}{3} \delta_{k l}\right]=\mathbf{0}
$$




$$
\begin{aligned}
\frac{\partial^{2} H_{2}}{\partial s_{k l} \partial s_{m n}}= & \frac{1}{2}\left(\frac{\partial}{\partial s_{m n}}+\frac{\partial}{\partial s_{n m}}\right) \frac{\partial H_{2}}{\partial s_{k l}} \\
& {\left[\begin{array}{llllll}
0 & -1 & -1 & 0 & 0 & 0 \\
-1 & 0 & -1 & 0 & 0 & 0 \\
-1 & -1 & 0 & 0 & 0 & 0 \\
0 & 0 & 0 & \frac{1}{2} & 0 & 0 \\
0 & 0 & 0 & 0 & \frac{1}{2} & 0 \\
0 & 0 & 0 & 0 & 0 & \frac{1}{2}
\end{array}\right] } \\
\frac{\partial^{2} H_{3}}{\partial s_{k l} \partial s_{m n}}= & \frac{1}{2}\left[\begin{array}{llllll}
0 & s_{z z} & s_{y y} & -s_{y z} & 0 & 0 \\
s_{z z} & 0 & s_{x x} & 0 & -s_{x z} & 0 \\
s_{y y} & s_{x x} & 0 & 0 & 0 & -s_{x y} \\
-s_{y z} & 0 & 0 & -\frac{1}{2} s_{x x} & \frac{1}{2} s_{x y} & \frac{1}{2} s_{x z} \\
0 & -s_{x z} & 0 & \frac{1}{2} s_{x y} & -\frac{1}{2} s_{y y} & \frac{1}{2} s_{y z} \\
0 & 0 & -s_{x y} & \frac{1}{2} s_{x z} & \frac{1}{2} s_{y z} & -\frac{1}{2} s_{z z}
\end{array}\right]
\end{aligned}
$$

d) Expressions for $\partial \theta / \partial H_{a}$

For sake of simplicity, the following is considered

$\theta=\arccos \left[\frac{H_{q}}{H_{p}^{3 / 2}}\right]=\arccos [C]$, with $C=\frac{H_{q}}{H_{p}^{3 / 2}}$

Thus, the derivative for $\partial \theta / \partial H_{a}$

$\frac{\partial \theta}{\partial H_{a}}=\frac{\partial \theta}{\partial H_{p}} \frac{\partial H_{p}}{\partial H_{a}}+\frac{\partial \theta}{\partial H_{q}} \frac{\partial H_{q}}{\partial H_{a}}$

The terms for the above expression are given by

$\frac{\partial \theta}{\partial H_{q}}=-\frac{1}{\sqrt{1-C^{2}}} \cdot \frac{1}{H_{p}^{3 / 2}}=K \cdot \frac{1}{H_{p}^{3 / 2}}$,

$\frac{\partial \theta}{\partial H_{p}}=-\frac{1}{\sqrt{1-C^{2}}} \cdot C \cdot\left(-\frac{3}{2} \frac{1}{H_{p}}\right)=K \cdot C \cdot\left(-\frac{3}{2} \frac{1}{H_{p}}\right)$,

$\frac{\partial H_{q}}{\partial H_{a}}=\left[\begin{array}{c}3 H_{1}^{2}+\frac{3}{2} H_{2} \\ \frac{3}{2} H_{1} \\ 1\end{array}\right]$,

$\frac{\partial H_{p}}{\partial H_{a}}=\left[\begin{array}{c}2 H_{1} \\ 1 \\ 0\end{array}\right]$,

with

$$
K=-\frac{1}{\sqrt{1-C^{2}}}
$$

e) Expressions for $\partial^{2} \theta / \partial H_{a} \partial H_{b}$

$$
\begin{aligned}
& \frac{\partial^{2} \theta}{\partial H_{a} \partial H_{b}}=\frac{\partial}{\partial H_{b}}\left(\frac{\partial \theta}{\partial H_{p}} \frac{\partial H_{p}}{\partial H_{a}}+\frac{\partial \theta}{\partial H_{q}} \frac{\partial H_{q}}{\partial H_{a}}\right)= \\
& =\left(\frac{\partial^{2} \theta}{\partial H_{p} \partial H_{p}} \frac{\partial H_{p}}{\partial H_{b}}+\frac{\partial^{2} \theta}{\partial H_{p} \partial H_{q}} \frac{\partial H_{q}}{\partial H_{b}}\right) \frac{\partial H_{p}}{\partial H_{a}}+\frac{\partial \theta}{\partial H_{p}}\left(\frac{\partial^{2} H_{p}}{\partial H_{a} \partial H_{b}}\right), \\
& \quad+\left(\frac{\partial^{2} \theta}{\partial H_{q} \partial H_{q}} \frac{\partial H_{q}}{\partial H_{b}}+\frac{\partial^{2} \theta}{\partial H_{q} \partial H_{p}} \frac{\partial H_{p}}{\partial H_{b}}\right) \frac{\partial H_{q}}{\partial H_{a}}+\frac{\partial \theta}{\partial H_{q}}\left(\frac{\partial^{2} H_{q}}{\partial H_{a} \partial H_{b}}\right)
\end{aligned}
$$

with

$$
\begin{aligned}
\frac{\partial^{2} H_{q}}{\partial H_{a} \partial H_{b}}=\left[\begin{array}{lll}
6 H_{1} & \frac{3}{2} & 0 \\
\frac{3}{2} & 0 & 0 \\
0 & 0 & 0
\end{array}\right] \text { and } \frac{\partial^{2} H_{p}}{\partial H_{a} \partial H_{b}}=\left[\begin{array}{lll}
2 & 0 & 0 \\
0 & 0 & 0 \\
0 & 0 & 0
\end{array}\right], \\
\frac{\partial^{2} \theta}{\partial H_{q} \partial H_{q}}=\frac{\partial K}{\partial H_{q}} \cdot\left(\frac{1}{H_{p}^{3 / 2}}\right) \\
\frac{\partial^{2} \theta}{\partial H_{q} \partial H_{p}}=\frac{\partial K}{\partial H_{p}} \cdot\left(\frac{1}{H_{p}^{3 / 2}}\right)+K \cdot\left(\frac{1}{H_{p}^{3 / 2}}\right) \cdot\left(-\frac{3}{2} \frac{1}{H_{p}}\right) \\
\frac{\partial^{2} \theta}{\partial H_{p} \partial H_{q}}=\frac{\partial K}{\partial H_{q}} \cdot C \cdot\left(-\frac{3}{2} \frac{1}{H_{p}}\right)+K \cdot \frac{\partial C}{\partial H_{q}} \cdot\left(-\frac{3}{2} \frac{1}{H_{p}}\right) \\
\frac{\partial^{2} \theta}{\partial H_{p} \partial H_{p}}=\frac{\partial K}{\partial H_{p}} \cdot C \cdot\left(-\frac{3}{2} \frac{1}{H_{p}}\right)+K \cdot \frac{\partial C}{\partial H_{p}} \cdot\left(-\frac{3}{2} \frac{1}{H_{p}}\right)-K \cdot C \cdot\left(-\frac{3}{2} \frac{1}{H_{p}}\right) \frac{1}{H_{p}}
\end{aligned}
$$

\section{A.4. Principal values}

The principal values are calculated by solving the characteristic equation

$\operatorname{det}(\mathbf{s}-s \mathbf{I})=0$

where $s$ represents the principal values of $\mathbf{s}$ and $\mathbf{s}$ is a tensor whose trace is zero,

$\mathbf{s}=\left[\begin{array}{lll}s_{x x} & s_{x y} & s_{x z} \\ s_{x y} & s_{y y} & s_{y z} \\ s_{x z} & s_{y z} & s_{z z}\end{array}\right]$.

$x, y$ and $z$ denote an orthogonal frame attached to the material (i.e. sheet rolling, transverse and normal directions, respectively).

This equation reduces to

$s^{3}-H_{1} s^{2}-3 H_{2} s-2 H_{3}=0$,

with

$H_{1}=\frac{1}{3} I_{1}=\frac{1}{3}\left(s_{x x}+s_{y y}+s_{z z}\right)$,

$H_{2}=\frac{1}{3} I_{2}=\frac{1}{3}\left(s_{y z}^{2}+s_{z x}^{2}+s_{x y}^{2}-s_{y y} s_{z z}-s_{z z} s_{x x}-s_{x x} s_{y y}\right)$,

$H_{3}=\frac{1}{2} I_{3}=\frac{1}{2}\left(2 s_{y z} s_{z x} s_{x y}+s_{x x} s_{y y} s_{z z}-s_{x x} s_{y z}^{2}-s_{y y} s_{z x}^{2}-s_{z z} s_{x y}^{2}\right)$.

Through a change of variables [8],

$s=s+H_{1}$,

the characteristic equation becomes

$\bar{s}^{3}-3 H_{p} \bar{s}-2 H_{q}=0$,

with

a) $H_{\mathrm{p}}=\mathrm{H}_{1}^{2}+\mathrm{H}_{2}$,

b) $H_{\mathrm{q}}=\frac{1}{2}\left(2 \mathrm{H}_{1}^{3}+3 \mathrm{H}_{1} \mathrm{H}_{2}+2 \mathrm{H}_{3}\right)$,

c) $\theta=\operatorname{arcos}\left[\frac{H_{\mathrm{q}}}{H_{\mathrm{p}}^{3 / 2}}\right]$,

Cardan's solutions to the characteristic equation are

a) $\bar{s}_{1}=z^{1 / 3}+\bar{z}^{1 / 3}$,

b) $\bar{s}_{2}=\omega z^{1 / 3}+\bar{\omega} \bar{z}^{1 / 3}$,

c) $\bar{s}_{3}=\bar{\omega} z^{1 / 3}+\omega \bar{z}^{1 / 3}$,

where $z$ is a complex number. $\omega$ is a complex constant $\left(\mathrm{e}^{-2 \mathrm{i} \pi / 3}\right)$, and $\bar{z}$ and $\bar{\omega}$ are their conjugate quantities. 
$z=H_{q}+\mathrm{i} \sqrt{H_{p}^{3}-H_{q}^{2}}$,

The principal values of $s$ can be written as

$\left\{\begin{array}{l}s_{1}=H_{1}+2 \sqrt{H_{1}^{2}+H_{2}} \cos (\theta / 3) \\ s_{2}=H_{1}+2 \sqrt{H_{1}^{2}+H_{2}} \cos \left(\frac{\theta+4 \pi}{3}\right), \\ s_{3}=H_{1}+2 \sqrt{H_{1}^{2}+H_{2}} \cos \left(\frac{\theta+2 \pi}{3}\right)\end{array}\right.$,

These values are ordered as [8]

$s_{1} \geq s_{2} \geq s_{3}, \quad s_{1}>s_{3}$.

\section{References}

[1] Cazacu O, Plunkett B, Barlat F. Orthotropic yield criterion for hexagonal closed packed metals. Int J Plast 2006;22(7):1171-94.

[2] Alves JL. An advanced constitutive model in the sheet metal forming simulation: the Teodosiu microstructural model and the Cazacu Barlat yield criterion. AIP Conf Proc 2004;712:1645-50.

[3] Cazacu O, Barlat F. Generalization of Drucker's yield criterion to orthotropy. Math Mech Solids 2001;6(6):613-30.

[4] Cazacu O. A criterion for description of anisotropy and yield differential effects in pressure-insensitive metals. Int J Plast 2004;20(11):2027-45.

[5] Plunkett B, Cazacu O, Barlat F. Orthotropic yield criteria for description of the anisotropy in tension and compression of sheet metals. Int J Plast 2008;24 (5):847-66.

[6] Plunkett B, Lebensohn RA, Cazacu O, Barlat F. Anisotropic yield function of hexagonal materials taking into account texture development and anisotropic hardening. Acta Mater 2006;54(16):4159-69.

[7] Yoon JW, Barlat F, Chung K, Pourboghrat F, Yang DY. Earing predictions based on asymmetric nonquadratic yield function. Int J Plast 2000;16(9):1075-104.

[8] Barlat F, Aretz H, Yoon JW, Karabin ME, Brem JC, Dick RE. Linear transfomation-based anisotropic yield functions. Int J Plast 2005;21(5):1009-39.

[9] Moayyedian F, Kadkhodayan M. Combination of modified Yld2000-2d and Yld2000-2d in anisotropic pressure dependent sheet metals, 1984; 2014. p. 2804-26.

[10] Banabic D. Sheet metal forming processes.Berlin Heidelberg: Springer-Verlag; 2010.

[11] Yoon J, Barlat F, Dick R, Karabin M. Prediction of six or eight ears in a drawn cup based on a new anisotropic yield function. Int J Plast 2006;22(1):174-93.

[12] Tritschler M, Butz A, Helm D, Falkinger G, Kiese J. Experimental analysis and modeling of the anisotropic response of titanium alloy Ti-X for quasi-static loading at room temperature. Int J Mater Form 2014;7(3):259-73.

[13] Tuninetti V, Gilles G, Milis O, Pardoen T, Habraken AM. Anisotropy and tension-compression asymmetry modeling of the room temperature plastic response of Ti-6Al-4V. Int J Plast 2015;67:53-68.

[14] Gilles G, Cazacu O, Hammami W, Habraken a M, Duchêne L. Experimental and numerical study of TA-6V mechanical behavior in different monotonic loading conditions at room temperature. Procedia IUTAM 2012;3:100-14.

[15] Revil-Baudard B, Cazacu O, Flater P, Kleiser G. Plastic deformation of highpurity $\alpha$-titanium: model development and validation using the Taylor cylinder impact test. Mech Mater 2014;80:264-75.

[16] Chandola N, Lebensohn RA, Cazacu O, Revil-Baudard B, Mishra RK, Barlat E. Combined effects of anisotropy and tension-compression asymmetry on the torsional response of AZ31 Mg. Int J Solids Struct 2015;58:190-200.

[17] Revil-Baudard B, Chandola N, Cazacu O, Barlat F. Correlation between swift effects and tension-compression asymmetry in various polycrystalline materials. J Mech Phys Solids 2014;70:104-15.

[18] Cazacu O, Revil-Baudard B, Barlat F. New interpretation of monotonic Swift effects: role of tension-compression asymmetry. Mech Mater 2013;57:42-52.

[19] Revil-Baudard B, Cazacu O. On the effect of the matrix tension-compression asymmetry on damage evolution in porous plastic solids. Eur J Mech A/Solids 2013;37:35-44.

[20] Alves JL, Cazacu O. Correlation between strength differential effects in the plastic flow of the matrix and the rate of damage growth in porous polycrystals. C. R. Méc. 2015;343(2):107-20.

[21] Cazacu O, Ionescu IR, Yoon JW. Orthotropic strain rate potential for the description of anisotropy in tension and compression of metals. Int J Plast 2010;26(6):887-904.

[22] Menezes LF, Teodosiu C. Three-dimensional numerical simulation of the deepdrawing process using solid finite elements. J Mater Process Technol 2000;97 $(1-3): 100-6$

[23] Oliveira MC, Alves JL, Menezes LF. Algorithms and strategies for treatment of large deformation frictional contact in the numerical simulation of deep drawing process. Arch Comput Methods Eng 2008;15(2):113-62.

[24] Teodosiu C. The plastic spin: microstructural origin and computational significance; 1989. p. 163-74.

[25] Simo JC, Taylor RL. Consistent tangent operators for rate-independent elastoplasticity. Comput Methods Appl Mech Eng 1985;48(1):101-18.

[26] Alves JL. Simulação numérica do processo de estampagem de chapas metálicas: modelação mecânica e métodos numéricos. University of Minho; 2003.

[27] Barlat F, Maeda Y, Chung K, Yanagawa M, Brem JC, Hayashida Y, Lege DJ, Matsui K, Murtha SJ, Hattori S, Becker RC, Makosey S. Yield function development for aluminum alloy sheets. J Mech Phys Solids 1997;45(11-12):172763.

[28] Barros PD, Oliveira MC, Alves JL, Andrade-Campos A, Menezes LF. Modelling of tension-compression asymmetry and orthotropic anisotropy in case of thin metallic sheets: identification procedure and case studies; 2015. p. 149-54.

[29] Barros PD, Neto DM, Alves JL, Oliveira MC, Menezes LF. DD3IMP, 3D fully implicit finite element solver: implementation of CB2001 yield criterion. Rom J Tech Sci - Appl Mech 2015;60(1-2):105-36.

[30] Banabic D, Aretz H, Comsa DS, Paraianu L. An improved analytical description of orthotropy in metallic sheets. Int J Plast . 2005;21(3):493-512.

[31] Kaschner G. Mechanical response of zirconium-II. Experimental and finite element analysis of bent beams. Acta Mater 2001;49(15):3097-108.

[32] Hughes TJR. Generalization of selective integration procedures to anisotropic and nonlinear media. Int J Numer Methods Eng 1980;15(9):1413-8. 